\title{
Évaluation du risque chimique dans l'environnement marin : exemple d'application aux installations industrielles du Nord-Cotentin
}

\author{
Chemical risk assessment in the marine environment: \\ example of the application to the industrial sector \\ in Nord-Cotentin
}

\author{
Michel Marchand, Chrystèle Tissier \\ Cellule Ifremer/Ineris d'Analyses des risques chimiques en milieu marin (ARC) \\ Centre Ifremer de Nantes, BP 21105, 44311 Nantes Cedex 3, France
}

\begin{abstract}
Résumé - La législation européenne sur les produits chimiques (substances existantes, substances nouvelles, biocides) impose une évaluation du risque chimique afin d'assurer la protection de la santé humaine et celle de l'environnement. Un document guide méthodologique européen, appelé « Technical Guidance Document » (TGD) a été élaboré en 1996 pour permettre la mise en œuvre d'une telle procédure qui soit commune à l'ensemble des États membres de l'Union européenne. Le concept méthodologique utilisé consiste à mettre en relation l'évaluation de la contamination du milieu, exprimée en concentration prévisible, baptisée PEC («Predicted Environmental Concentration») et l'évaluation des effets, exprimée en terme de concentration prévisible sans effets, baptisée PNEC («Predicted No-Effect Concentration »). La caractérisation du risque est basée sur la valeur du rapport PEC/PNEC.

La révision du document guide européen a conduit à scinder la procédure d'évaluation du risque environnemental dans les milieux aquatiques en deux chapitres distincts, l'un concernant le milieu continental, le second spécifiquement le milieu marin. Ce travail d'adaptation du TGD au milieu marin a été mené durant deux ans dans le cadre d'une collaboration entre la Commission européenne et la Convention OSPAR. La première version de ce document constitue un document de référence pour l'évaluation du risque chimique en milieu marin. Sans remettre en question le concept méthodologique de base, les modifications apportées pour adapter le TGD au milieu marin concernent : le comportement des substances, la biodégradation, l'évaluation de l'exposition et les scénarios d'émission envisagés à l'échelle locale et régionale, l'évaluation des effets, l'évaluation de l'empoisonnement secondaire chez les prédateurs via le transfert trophique et la protection du milieu marin vis-à-vis des substances PBT (persistantes, bioaccumulables et toxiques).

Dans le cadre de l'évaluation des risques environnementaux des rejets chimiques des installations nucléaires du Nord-Cotentin, cette méthodologie a été utilisée sur 27 substances parmi lesquelles quelques organiques et un grand nombre de métaux. Les premiers résultats de cette étude mettent en évidence les limites et les incertitudes de la méthode notamment dans la détermination des PNEC en milieu marin où les données écotoxicologiques sont faibles.
\end{abstract}

Mots clés - risque chimique, milieu marin, TGD 


\begin{abstract}
In the context of the European legislation on chemicals (existing, new chemicals and biocides) a risk assessment for the environment and human health is performed. A technical guidance document (TGD), in support of Commission Directive 93/67/EEC on risk assessment for new notified substances and Commission Regulation (EC) No1488/64 on risk assessment for existing substances has been developed in 1996 and was recently adapted for the marine environment. The methodological concept is based on the relation between a value representing the contamination level of a chemical substance in the environment (exposure assessment) and a value representing its effects on the environment (effects assessment). The exposure value is expressed as PEC for "Predicted Environmental Concentration" and the effect value is defined as the PNEC, for "Predictive No effect Concentration". The risk characterisation is represented by the value of the PEC/PNEC ratio.

The recent adaptation of the technical guidance document lead to two different aquatic assessments: one for the inland compartment and one for the marine compartment. This adaptation was conducted during two years and is the result of the co-operation of the European Commission and the OSPAR Convention. The main adaptations of the document to the marine environment concern the dilution rate in the water compartment, the behaviour of the substances, biodegradation, the local and regional exposure assessment scenario, effects assessment and secondary poisoning. A specific part dealing with PBT substances (Persistent, Bioaccumulable and Toxic) was also added. The initial concept of the guidance was however the same as for the inland environment.

The document was used in the context of a specific study in France: "Environmental risk assessment of chemical releases of nuclear installations in Nord-Cotentin, France". The chemical releases of industrial sites were mainly composed of metals and a few organic chemicals. The releases of 27 substances in the marine environment were assessed. Limits and uncertainties of the methodology were underlined regarding, in particular, the determination of PNEC and the lack of ecotoxicological data for the marine environment.
\end{abstract}

Key words - chemical risk assessment, marine environment, TGD

Le chapitre 19 de l'Agenda 21 de la Conférence de Rio en 1992 a fixé les grands principes d'une nouvelle politique de gestion des produits chimiques et a inspiré la politique actuelle de réduction et d'élimination des substances chimiques dangereuses de l'environnement. Les principales recommandations que l'on peut retenir sont les suivantes :

- la nécessité d'une harmonisation internationale de la classification et de l'étiquetage des produits chimiques. Ce travail a été confié à l'OCDE et s'est terminé fin 1998 (http: //www . oecd.org);

- l'encouragement d'une large circulation de l'information. Depuis juin 2001, l'ensemble des informations sur les produits chimiques recensé par les principales organisations internationales (OIT, OMS, FAO, PNUE, etc.) est accessible gratuitement sur le Net (http: //www . inchem.org);

- la prévention du trafic illégal des substances toxiques et produits dangereux;

- l'obligation d'évaluer les risques des substances chimiques (existantes ou nouvelles, biocides, substances phytopharmaceutiques);

- le renforcement des capacités nationales de surveillance et de gestion. Ainsi, en 2001, I'Institut français de recherche pour l'exploitation de la mer (Ifremer) et l'Institut national de l'environnement industriel et des risques (Ineris) ont décidé de mettre en commun 
leurs compétences respectives afin d'améliorer la capacité française d'expertise dans le domaine de l'évaluation du risque chimique en milieu marin en créant une Cellule opérationnelle mixte, dénommée ARC qui est localisée au Centre Ifremer de Nantes;

\section{UNE PROCÉDURE AU SERVICE D'UNE LÉGISLATION}

La législation européenne sur les produits chimiques impose une évaluation du risque chimique des substances mises sur le marché afin d'assurer une protection de l'homme et de l'environnement. L'évaluation du risque concerne les substances existantes, c'est-à-dire les substances mises sur le marché européen entre 1971 et 1981 (Règlement CEE $\left.n^{\circ} 793 / 93\right)$, les substances nouvelles commercialisées après 1981 (Directive 93/67/CEE) et plus récemment les biocides (Directive 98/8/CE). Les principes directeurs de l'évaluation du risque chimique sont définis dans le Règlement (CE) n 1488/94 et sont détaillés dans le guide méthodologique européen, le «Technical Guidance Document » (TGD) (CE, 1996). Ce document de référence permet la mise en œuvre d'une procédure qui soit commune à l'ensemble des États membres de l'Union européenne. La gestion technique de la législation européenne sur les substances chimiques est assurée au Centre commun de recherche de la Commission européenne (Ispra, Italie) par le Bureau européen des substances chimiques ("European Chemicals Bureau »-ECB, http: //ecb.ei.jrc.it).
Le principe de l'évaluation des risques chimiques dans l'environnement est basé sur la relation entre les effets indésirables d'une substance sur des organismes représentatifs d'un milieu considéré et son niveau de contamination connue (c'est-à-dire mesuré) ou raisonnablement prévisible (c'est-àdire calculé à l'aide de modèles appropriés) dans l'environnement. II est estimé par cette approche que la protection des espèces, dont la diversité constitue la structure d'un écosystème, permet de protèger le fonctionnement de l'écosystème lui-même. La caractérisation du risque procède en 4 étapes :

- l'évaluation des dangers repose sur la connaissance des propriétés intrinsèques des substances chimiques;

- l'évaluation de l'exposition vise à estimer les niveaux de contamination d'une substance chimique dans les différents compartiments environnementaux; il s'agit d'obtenir des concentrations prévisibles, appelées PEC («Predicted Environmental Concentration ») à partir de données mesurées dans l'environnement ou de valeurs calculées à l'aide de modèles ;

- l'évaluation des effets consiste à définir la relation concentrationseffets, le résultat attendu est la détermination de concentrations sans effets prévisibles, dites PNEC («Predicted No Effect Concentration ») qui sont déduites de données écotoxicologiques sur des espèces représentatives, soit de toxicité aiguë $\left(\mathrm{L}(\mathrm{E}) \mathrm{C}_{50}\right.$, «lethal(effect) concentration ») ou chronique (NOEC « no-observed effect concentration »); 
- la caractérisation du risque par la mise en relation de l'exposition et des effets potentiels, c'est-à-dire par le rapport PEC/PNEC, selon qu'il est inférieur à 1 (absence de risque) ou supérieur à 1 (risques réels ou potentiels).

\section{PRINCIPES DIRECTEURS DU GUIDE MÉTHODOLOGIQUE EUROPÉEN (TGD) POUR LES MILIEUX AQUATIQUES}

Le guide méthodologique européen (TGD) constitue un document qui offre une méthodologie suffisamment précise et détaillée pour évaluer le risque d'une substance chimique vis-à-vis de la santé humaine et de l'environnement. La première version de ce document (CE, 1996) définit plusieurs cibles privilégiées pour la protection de l'environnement : les milieux aquatiques, les sols, l'atmosphère, la faune sauvage représentée par les grands prédateurs (oiseaux et mammifères) et le fonctionnement des stations d'épuration. L'évaluation du risque chimique dans l'environnement est réalisée à deux échelles spatiales : une échelle locale proche d'un point de rejet et une échelle régionale plus étendue qui intègre les apports de contamination multiples ponctuels et diffus. La caractérisation du risque chimique est réalisée à ces deux échelles en comparant les valeurs du rapport PEC/PNEC évoqué précédemment. Nous limiterons la présentation du TGD aux seuls milieux aquatiques qui nous intéressent. Pour chacun des deux scénarios, local et régional évoqués précédemment, la caractérisation du risque chimique concerne :

- le milieu aquatique proprement dit (PEC/PNEC eau) ;

- le milieu sédimentaire (PEC/PNEC sédiment);

- les prédateurs supérieurs de la faune sauvage pour lesquels l'impact est évalué selon la capacité de transfert de la substance vers les niveaux trophiques supérieurs, via la chaîne alimentaire.

\section{1 Évaluation de l'exposition}

La contamination du milieu peut être estimée, soit par des valeurs mesurées (ce qui nécessite une interrogation sur la représentativité, la précision et l'exactitude des données), soit par des valeurs calculées (ce qui pose la question de la fiabilité des modèles utilisés). Pour les milieux aquatiques, l'évaluation de l'exposition prend en compte l'estimation des émissions, la dilution de l'effluent dans le milieu récepteur, les processus de distribution entre les différents compartiments (eau, sédiment, biota) en utilisant les coefficients de partage et les différents processus d'élimination (volatilisation, hydrolyse, photolyse, biodégradation). II convient de préciser ici que le TGD est essentiellement dédié aux substances organiques apolaires. Les substances organiques ionisables, les métaux ainsi que les mélanges (ex. hydrocarbures) font l'objet d'annexes pour souligner certaines spécificités.

Les valeurs d'exposition sont déterminées à l'échelle locale qui prend en considération l'impact majeur d'un rejet ponctuel dans un champ environnemental proche et l'échelle régionale 
qui permet de déterminer un niveau de contamination de base (bruit de fond) lié à des apports contaminants multiples et divers.

Échelle locale. La concentration dans l'eau à l'échelle locale est calculée après mélange complet de l'effluent sortant d'une station d'épuration dans le milieu récepteur. Le processus de dispersion majeur pris en compte est la dilution; la dégradation, la volatilisation, la sédimentation ne sont pas considérés à cette échelle de temps réduite. Un facteur de dilution standard est utilisé, malgré la grande variabilité des situations qui peuvent être rencontrées, le facteur de dilution pouvant varier de 1 (rejet dans le lit d'un cours d'eau sec) à plus de 100000 . Le facteur de dilution standard choisi par défaut est de 10.

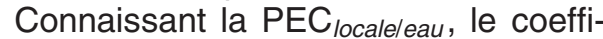
cient de partage $\mathrm{K}_{\mathrm{MES} / \mathrm{eau}}$ et la concentration des matières en suspension (MES), il est possible de déterminer la valeur $\mathrm{PEC}_{\text {locale/sédiment }}$.

Échelle régionale. Un environnement régional standard est défini par un espace de $200 \times 200 \mathrm{~km}$, occupé par 20 millions d'habitants et où la production de la substance chimique étudiée est choisie arbitrairement à $10 \%$ de la production européenne. Le niveau de contamination de la substance au niveau régional est calculé en utilisant un modèle multi-compartiments environnementaux, de type Mackay, basé sur le concept de fugacité (Mackay et al., 1992). Sur la base des propriétés de la substance et des paramètres environnementaux standards adoptés dans le modèle, des $P E C_{\text {régional sont déterminées dans les }}$ différents compartiments (eau, sols, air). Les principales caractéristiques qui définissent cet environnement générique régional sont la surface $(200 \times$ $200 \mathrm{~km})$, la répartition des milieux, eau (3\%), sols naturels (60\%), sols cultivés ( $27 \%$ ), milieu artificialisé urbain et industriel (10\%), colonne d'air (1 km), colonne d'eau (3 m), profondeur du sédiment $(3 \mathrm{~cm})$, fraction du sédiment en aérobiose (10\%), précipitation annuelle $(700 \mathrm{~mm})$, vitesse du vent $(3 \mathrm{~m} / \mathrm{s})$, temps de résidence de l'air ( 0,7 jour), de l'eau (40 jours), ...

La qualité de la valeur prédite nécessite une bonne connaissance du comportement de la substance (pour éviter les valeurs par défaut) et un bon ajustement des hypothèses retenues. Les valeurs par défaut sont en règle générale conservatives et conduisent à une surestimation de l'exposition, pouvant induire des actions de réduction des risques coûteuses et inappropriées. À l'inverse, une sous-estimation de l'exposition peut conduire à une sous-estimation du risque environnemental. II est admis que la partie la plus incertaine dans la procédure de l'évaluation du risque chimique concerne l'évaluation des émissions et pertes (facteurs d'incertitude allant de 10 à 1000) et surtout l'évaluation de l'exposition (facteur d'incertitude pouvant aller de 10 à 10000). À titre d'exemple, les hypothèses retenues pour estimer une valeur calculée (PEC) de deux substances biocides présentes dans les peintures antisalissures utilisées sur des bateaux de plaisance aboutissent à de fortes surestimations par rapport aux valeurs mesurées dans les eaux côtières britanniques, d'un facteur 26 à 
1350 pour l'Irgarol 1051 et d'un facteur 14 à 290 pour le diuron (Thomas et al., 2001).

\section{2 Évaluation des effets}

L'évaluation des effets est basée sur les données en écotoxicologie, toxicité aiguë (généralement touchant à la survie de l'espèce, $C L(E)_{50}$ ) et/ou toxicité chronique (généralement exprimée en terme de concentration sans effet observé, "no observed effect concentration », NOEC). Cette approche écotoxicologique qui est opérationnelle (standardisation de tests) est justifiée par l'hypothèse de départ qu'en protégeant les espèces les plus sensibles, la structure d'un écosystème est protégée et par conséquent son fonctionnement. L'objectif est d'estimer la concentration d'une substance chimique ne provoquant aucun effet adverse sur l'écosystème aquatique, la PNEC («Predicted no-effect concentration »). Pour cela deux méthodes sont possibles selon le jeu de données disponibles, soit la méthode par facteur d'extrapolation lorsque le nombre de données disponibles est limité, soit la méthode par extrapolation statistique lorsque le nombre de données disponibles est plus important.

Méthode par facteur d'extrapolation. Pour les milieux aquatiques, trois espèces sont sélectionnées pour représenter au minimum trois niveaux trophiques représentatifs : une algue, un crustacé (généralement la daphnie) et un poisson; ceci constitue le « jeu de données de base » pour l'évaluation des effets. Selon la nature des données disponibles, différents facteurs d'extrapolation empiriques sont appliqués pour évaluer la concentration sans effet prévisible (PNEC), allant de 1000 (si l'on ne dispose que de données de toxicité aiguë pour les trois espèces) à 10 (si l'on dispose de données de toxicité chronique), voire à un jugement expert au cas par cas si les données ont été obtenues sur le terrain (Tab. 1). Ces facteurs d'extrapolation sont appliqués sur les données de toxicité de l'espèce la plus sensible. Ils prennent en compte :

- les variations intra-spécifiques liées à l'état physiologique des individus d'une même espèce ;

- les variations inter-spécifiques résultant des différences de sensibilité entre les différentes espèces de l'écosystème vis-à-vis d'une même substance;

- les extrapolations de la toxicité à court-terme vers le long-terme. Des effets sublétaux, non détectables à court-terme, peuvent apparaître à long-terme et mettre en danger une population;

- les extrapolations des données de laboratoire qui ne tiennent pas compte de l'état initial de l'écosystème. Par ailleurs, des effets additifs, synergiques, ou antagonistes dus à la présence d'autres substances dans le milieu peuvent modifier les effets de la substance testée sur la biocénose;

- les variations entre les différents laboratoires et expérimentateurs réalisant les essais.

Méthode par extrapolation statistique. Si un grand nombre de données de toxicité à long terme est disponible (plus de 10 à 15 NOEC) pour des espèces différentes appartenant au 
Tableau 1. Facteurs d'extrapolation utilisés pour évaluer la concentration sans effet prévisible (PNEC) d'une substance chimique dans les milieux aquatiques.

Table 1. Assessment factors proposed for deriving a Predicted No Effect Concentration (PNEC) for chemical substances in the aquatic environment.

\begin{tabular}{|l|c|}
\hline & $\begin{array}{c}\text { Facteur } \\
\text { d'extrapolation }\end{array}$ \\
\hline $\begin{array}{l}\text { Au moins une donnée de toxicité à court terme (C(E)L50) pour chacun des } \\
\text { trois niveaux trophiques du dossier de base }\end{array}$ & 1000 \\
\hline $\begin{array}{l}\text { Une NOEC chronique (poissons ou daphnies ou un organisme } \\
\text { représentatif des eaux salines) }\end{array}$ & 100 \\
\hline $\begin{array}{l}\text { Deux NOEC chroniques pour les espèces représentant deux niveaux } \\
\text { trophiques (poissons et/ou daphnies ou un organisme représentatif des } \\
\text { eaux salines et/ou algues) }\end{array}$ & 50 \\
\hline $\begin{array}{l}\text { NOEC chroniques pour au moins trois espèces (normalement } \\
\text { poissons, daphnies ou un organisme représentatif des eaux salines et } \\
\text { algues) représentant trois niveaux trophiques }\end{array}$ & 10 \\
\hline $\begin{array}{l}\text { Autres cas, y compris les données obtenues sur le terrain ou } \\
\text { écosystèmes modèles, qui permettent de calculer et d'appliquer des fac- } \\
\text { teurs de sécurité plus précis }\end{array}$ & $\begin{array}{c}\text { Évaluation par cas } \\
\text { cas }\end{array}$ \\
\hline
\end{tabular}

moins à 8 groupes taxonomiques, le degré d'incertitude qui prévalait précédemment diminue. Pour améliorer l'estimation de la PNEC, il est alors possible d'utiliser une méthode d'extrapolation statistique qui consiste à déterminer le $5^{\mathrm{e}}$ percentile $\left(\mathrm{HC}_{5}{ }^{(1)}\right)$ de la distribution des données NOEC, ce qui revient à évaluer la concentration qui protège $95 \%$ des espèces représentées (Fig. 1). La PNEC est déterminée en appliquant à la valeur $\mathrm{HC}_{5}$ un facteur d'extrapolation choisi entre 1 et 5 selon un jugement expert défini au cas par cas, ce qui permet d'introduire l'incertitude due à la variabilité biologique et à l'extrapolation statistique. Cette méthode suppose que les NOECs observées sur les différentes espèces suivent une

(1) $\mathrm{HC}_{5}=$ Hazardous Concentration , concentration provoquant des effets écotoxicologiques pour seulement $5 \%$ des espèces représentées dans le jeu de données étudié. fonction de distribution, le groupe d'espèces testées dans le laboratoire est un échantillon aléatoire de cette distribution. Différentes fonctions de distribution peuvent être employées, par exemple la distribution log-logisitique (Aldenberg et Slob, 1993) ou la distribution log-normale (Aldenberg et Jaworska, 2000). Les méthodes d'extrapolation statistique s'appliquent à des NOECs provenant d'études chroniques, de préférence sur la durée de vie totale de l'espèce ou sur des tests sur plusieurs générations. De plus, Iorsque plusieurs NOECs sont observées sur une même espèce, c'est la moyenne géométrique de ces NOEC qui est utilisée. Selon le TGD, I'utilisation de l'extrapolation statistique requiert au minimum les groupes taxonomiques suivants :

- poissons (les espèces habituellement testées comme le saumon Salmo salar); 
- une autre famille de Cordés (poisson, amphibien, etc.) ;

- un crustacé (cladocère, copépode, ostracode, isopode, amphipode...);

- un insecte (moustique, libellule. . .);

- un autre phylum que les Arthropodes ou les Cordés (rotifères, annélides, mollusques...);

- des algues (phytoplancton en général) ;

- des plantes supérieures.

Les points suivants doivent être pris en compte pour estimer la valeur du facteur d'extrapolation :

- la qualité globale du jeu de données et les critères d'effets correspondants;

- si les tests sont véritablement chroniques selon leur durée d'exposition;

- la diversité et la représentativité des groupes taxonomiques, et les différences dues au stade du cycle de vie, stratégies alimentaires et les niveaux trophiques des organismes étudiés;

- les connaissances sur le mode d'action du contaminant (avec l'exposition à long-terme) ;

- les incertitudes dues à l'utilisation de l'extrapolation statistique autour $\mathrm{du} 5^{\mathrm{e}}$ percentile : intervalle de confiance à $50 \%$ et à $95 \%$;

- comparaisons avec des études de terrain et de mésocosmes (multiespèces) si existantes, afin d'évaluer l'extrapolation des résultats entre le laboratoire et le terrain.

Les valeurs de NOEC en dessous de $5 \%$ de la $\mathrm{HC}_{5}$ doivent être discutées, car si elles proviennent d'un même niveau trophique, cela peut être l'indication de l'existence d'un groupe particulièrement sensible impliquant que certaines des hypothèses faites pour appliquer cette méthode peuvent ne pas avoir été rencontrées.

L'approche par l'extrapolation statistique est actuellement toujours en discussion et nécessite des validations complémentaires. Son principal avantage réside dans le fait qu'elle utilise l'entière distribution de sensibilité des espèces dans un écosystème pour dériver une PNEC, au lieu de prendre simplement la plus faible NOEC. Ses inconvénients souvent mis en avant sont le manque de transparence par rapport aux méthodes standardisées, la comparabilité des différents critères d'effets, ou encore le choix arbitraire d'un percentile spécifique de $5 \%$.

\subsection{Commentaires}

En dépit d'une procédure d'évaluation du risque très simplifiée (rapport PEC/PNEC), la caractérisation du risque chimique est souvent rendue difficile par l'absence de données. À titre d'exemple, une étude menée sur plusieurs estuaires britanniques a montré que sur 71 substances identifiées, les critères d'effets (PNEC) n'ont pu être estimés que pour $68 \%$ d'entre elles (Matthiessen et al., 1993). Par ailleurs l'examen des bases de données en écotoxicologie montre un très large déficit en données. Sur 51400 enregistrements de la base AQUIRE de l'Agence pour la Protection de l'Environnement (EPA) aux États-Unis, $68 \%$ des données concernent des résultats de toxicité aiguë et seulement $4 \%$ des résultats de toxicité chronique. La base de données de l'European Centre for 


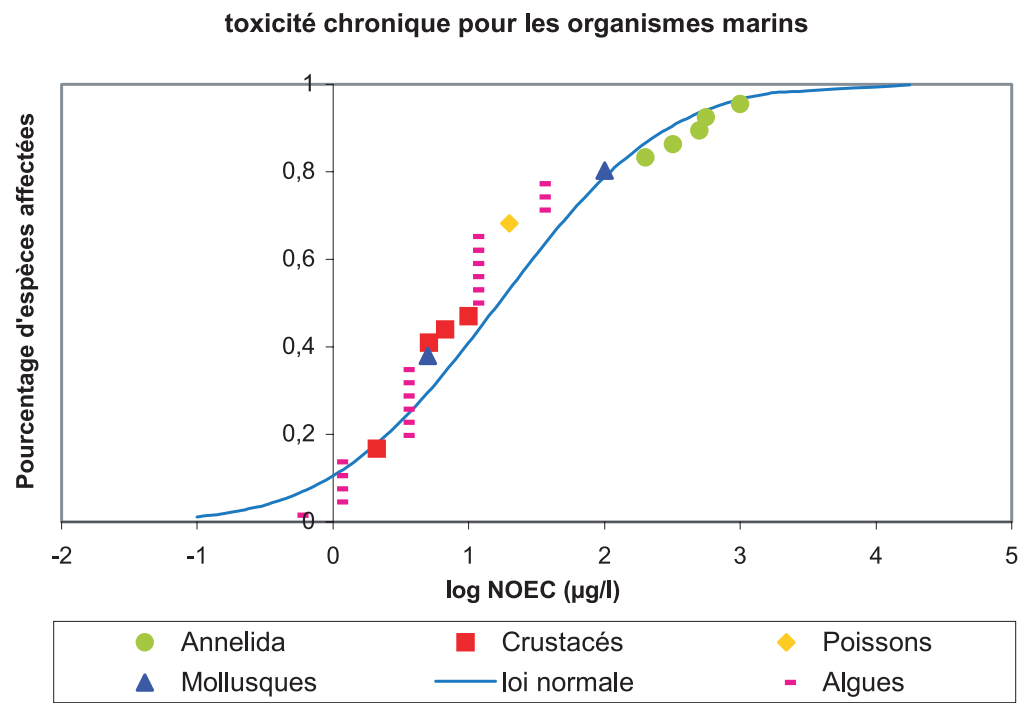

Fig. 1. Distribution de la toxicité chronique du cadmium vis-à-vis des organismes marins. La valeur $\mathrm{HC}_{5}(50 \%)=0,424 \mu \mathrm{g} / \mathrm{l}$ avec I.C $\left(5 \% \_95 \%\right)=[0,147 ; 0,960]$. Les données sur organismes marins disponibles sont faibles notamment pour les poissons pour lesquels seule une étude est disponible. L'ensemble des données algues proviennent d'une seule et même étude et certains groupes spécifiques du milieu marin ne sont pas représentés (Echinodermes). Par conséquent, un facteur de sécurité de 5 est appliqué à la valeur de la HC5, d'où $P N E C_{\text {eau }}=0,424 / 5=0,085 \mu \mathbf{g ~ C d / l ~ = ~} 85 \mathbf{~ n g} / \mathbf{l}$.

Fig. 1. Distribution of cadmium chronic toxicity data for marine organisms.

Ecotoxicology and Toxicology of Chemicals (ECETOC) de l'industrie chimique européenne (CEFIC) indique que sur 2200 enregistrements, $85 \%$ concernent des tests de toxicité réalisé sur des espèces d'eaux douces et seulement $15 \%$ sur des espèces d'eaux marines et estuariennes (ECETOC, 2001). L'examen des données écotoxicologiques existantes entre les espèces vivant en eaux douces et celles vivant dans les eaux salines suggèrent une corrélation raisonnable, du moins pour les tests de toxicité aiguë (Hutchinson et al., 1998). II ne semble pas y avoir de différences de sensibilité significatives entre les espèces d'eaux douces et d'eaux salines. Lorsque les tests ont été réalisés avec des critères d'effets (survie, croissance ou reproduction) et des espèces comparables (crustacés, poissons), les différences de sensibilité entre les valeurs observées se situent dans un facteur 2 à 10 au maximum. Des différences plus grandes, supérieures au facteur 10, ont toutefois pu être montrées, pour des espèces appartenant à des niveaux trophiques analogues, pour plusieurs métaux et pesticides. Enfin, si de nombreuses substances, prises individuellement, sont présentes en deçà des critères écotoxicologiques, elles 
peuvent également exercer, en terme de mélanges, des effets sur les organismes sans qu'il soit permis actuellement d'en préciser la quantification.

Le guide méthodologique européen (TGD), initialement développé pour évaluer les risques chimiques qui peuvent apparaître en premier lieu dans l'environnement continental terrestre et aquatique, n'était pas réellement utilisable en l'état pour le milieu marin. La révision récente de ce document a amené à consacrer un chapitre spécifique pour le milieu marin. Ce travail d'adaptation du TGD au milieu marin a été mené durant deux ans dans le cadre d'une collaboration entre I'Union européenne (révision du TGD) et la Convention OSPAR (définition d'un outil dans le cadre de la stratégie développée sur les substances dangereuses) (http://www.ospar.org). Ce document qui a été finalisé en 2002 (ECB, 2002) constitue un premier guide méthodologique de référence à l'échelle européenne pour évaluer les risques des substances chimiques dans le milieu marin.

\section{ADAPTATION DU GUIDE MÉTHODOLOGIQUE EUROPÉEN (TGD) POUR LE MILIEU MARIN}

Le milieu marin regroupe les zones estuariennes, le milieu côtier et la haute mer. Le concept méthodologique d'évaluation du risque chimique, c'est-à-dire l'évaluation du rapport PEC/PNEC, est conservé. Trois « cibles » sont à protéger : les espèces du milieu pélagique, celles du milieu benthique et la faune sauvage (oiseaux, mammifères, ....). Les modifications qui ont été apportées pour tenir compte des spécificités du milieu marin ont été les suivantes : la dispersion du rejet dans le champ proche, le comportement des substances au sein des différents compartiments environnementaux, la biodégradation, les scénarios d'émission à l'échelle locale et régionale pour évaluer l'exposition, l'évaluation des effets, l'évaluation de l'intoxication secondaire chez les grands prédateurs via le transfert trophique, le cas du milieu hauturier.

\subsection{Rejet et dispersion physique}

Au niveau du champ proche, il a été admis que la dilution d'un rejet est plus importante dans les eaux côtières que dans les eaux fluviales (Lewis, 1997), du fait des différences de salinité et de l'influence des courants de marée. Un taux de dilution générique de 100 a été adopté au lieu de 10 pour les eaux continentales.

\subsection{Distribution des substances dans l'environnement}

Rappelons que la procédure décrite dans le TGD concerne fondamentalement les substances organiques non polaires, les substances organiques ionisables et les métaux font l'objet de commentaires spécifiques en annexe du document. Si l'effet de sel (" salting-out») joue sur la solubilité des substances et donc sur les coefficients de partage (air/eau, sédiment/eau, biota/eau), la réduction de la solubilité dans l'eau de mer par rapport aux eaux douces, en moyenne d'un facteur 1,36 (Xie et al., 1997) n'est pas suffisamment significative, compte 
Tableau 2. Valeurs de demi-vie des substances par défaut pour l'évaluation du risque chimique en milieu marin.

Table 2. Default half life values for marine risk assessment of chemical substances.

\begin{tabular}{|l|c|c|c|}
\hline $\begin{array}{l}\text { Biodégradabilité } \\
\text { de la substance }\end{array}$ & Eau douce & Estuaire & Milieu marin \\
\hline Facile & 15 jours & 15 jours & 50 jours \\
\hline Facile au-delà de 10 jours & 50 jours & 50 jours & 150 jours \\
\hline Inhérente & 150 jours & 150 jours & $\infty$ \\
\hline Substance persistante & $\infty$ & $\infty$ & $\infty$ \\
\hline
\end{tabular}

tenu des degrés d'incertitudes dans la démarche de l'analyse du risque. II est donc admis de pouvoir prendre en considération les données de solubilité des substances obtenues en eau douce. La stabilité du pH de l'eau de mer (proche de 8) ne modifie pas les remarques initiales du TGD sur les taux de dissociation des substances organiques ionisables (fonction du pKa des substances). Par contre, il est fortement recommandé de voir au cas par cas le comportement des métaux dans les eaux marines, compte tenu de l'influence très importante de la composition de l'eau de mer sur la spéciation d'un élément, donc sur sa solubilité, ses coefficients de partage et sa biodisponibilité.

\subsection{Biodégradation}

Les données existantes sur l'estimation de la demi-vie des substances chimiques en milieu marin ne sont pas nombreuses. Prenant en considération une densité des communautés bactériennes plus faibles dans les eaux marines, il a été admis, en première approche, que la durée de vie d'une substance chimique en milieu marin serait trois fois plus importante que dans les eaux fluviales. Les cinétiques de dégradation dans les eaux estua- riennes ont été adoptées comme analogues à celles prises pour les eaux fluviales. Le tableau 2 préconise, en absence de données expérimentales, l'utilisation des valeurs de demi-vie suivantes sur la base des résultats de tests standardisés de biodégradabilité. II est donc admis que la durée de vie d'une substance chimique en eau marine est au minimun trois fois plus longue qu'en eau douce.

\section{4 Évaluation de l'exposition au niveau local et régional}

Nous avons vu précédemment que le niveau d'exposition, exprimé par l'estimation de la concentration prévisible environnementale (PEC), s'exprime selon le compartiment aquatique concerné (eau, sédiment) et à deux échelles, locale et régionale.

Le scénario local pour le milieu marin conserve les mêmes caractéristiques de base que celles adoptées en milieu continental, à la seule différence du facteur de dilution pris à 100 au lieu de 10 pour un rejet en eaux côtières. Les matières en suspension dans l'eau de mer sont prises à $15 \mathrm{mg} / \mathrm{l}$.

Le scénario régional initialement adopté pour le milieu continental (zone de $20 \times 200 \mathrm{~km}$ représentant $97 \%$ de sols et $3 \%$ d'eau) n'est pas utilisable 


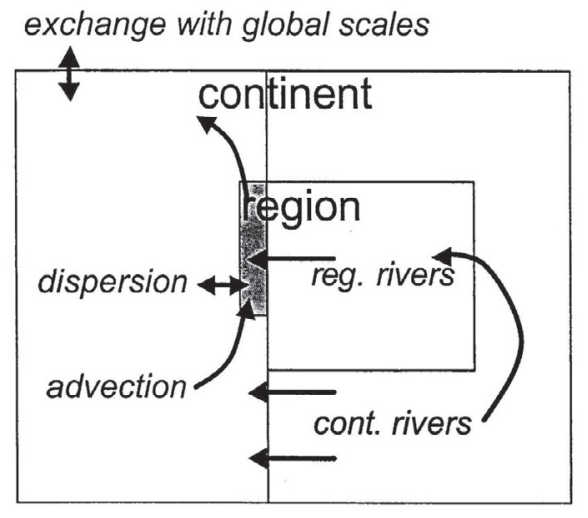

Fig. 2. Scénario régional marin (ECB, 2002).

Fig. 2. Regional sea scenario.

pour le milieu marin. Un scénario régional spécifique a été défini, constitué par une zone côtière de $40 \mathrm{~km}$ de long, $10 \mathrm{~km}$ de large, $10 \mathrm{~m}$ de profondeur avec une charge particulaire de $5 \mathrm{mg} / \mathrm{l}$ et un taux de dispersion de $50 \mathrm{~m}^{2} / \mathrm{s}$ (Fig. 2). La zone régionale marine ainsi définie reçoit les apports fluviaux $\left(1000 \mathrm{~m}^{3} / \mathrm{s}\right)$ du compartiment continental régional $(200 \times 200 \mathrm{~km})$ et $1 \%$ des rejets directs de la substance étudiée; elle est en échange permanent avec le milieu marin du large par advection (courant marin de $3 \mathrm{~cm} / \mathrm{s}$ ). Ceci représente sensiblement un scénario analogue au panache des eaux du Rhin et de la Meuse jusqu'à 10 km de la côte.

On remarquera que dans cette présentation, aucune condition spécifique n'est proposée, ni à une échelle locale, ni régionale, pour prendre en compte la situation particulière des estuaires. II est considéré dans une première approche que les estuaires seront protégés, du fait de leur situation intermédiaire, soit par l'évaluation du risque en milieu continental (fleuves), soit en milieu marin (eaux côtières).

\section{5 Évaluation des effets}

Pour les écosystèmes marins, une PNEC peut être extrapolée à partir de tests mono-spécifiques réalisés sur des espèces d'eaux douces et/ou marines) et de l'application de facteurs d'extrapolation. Le choix de ces facteurs dans la nouvelle version du TGD adapté aux spécificités du milieu marin a été guidé par plusieurs réflexions, conduisant à modifier, pour le milieu marin, dans le sens d'une plus grande précaution, les facteurs d'extrapolation déjà utilisés pour les milieux aquatiques continentaux. Le premier constat concerne la pauvreté des données écotoxicologiques marines actuellement disponibles par rapport à celles des eaux douces (ECETOC, 2001). De ce fait, les données eaux douces sont généralement prises comme référence dans la procédure d'évaluation du risque chimique en milieu marin. Elles ne peuvent refléter la grande biodiversité marine, d'où la nécessité de disposer de données écotoxicologiques sur un plus grand nombre de taxons. Paradoxalement, certains domaines marins génériques (estuaires) ou géographiques (mer Baltique) présentent une diversité d'espèces limitée; des effets néfastes sur certaines d'entre elles et pour lesquelles l'information est manquante peuvent avoir des conséquences inattendues sur ces écosystèmes spécifiques. Ces arguments, loin d'être opposables, concourent au choix d'une plus grande précaution à appliquer au domaine 
marin. Par ailleurs, il est reconnu que le milieu marin constitue le récepteur ultime des rejets contaminants dans les milieux aquatiques et les effets à long terme des contaminants chimiques sont difficilement prévisibles dans l'état actuel de nos connaissances.

L'ensemble de ces considérations a donc amené à conserver, sur la base de données en écotoxicologie aiguë, le même facteur d'extrapolation de 1000 à condition de disposer de données sur deux groupes taxonomiques marins supplémentaires (échinodermes et mollusques par exemple), sinon de prendre une précaution supplémentaire avec un facteur d'extrapolation de 10000 pour évaluer la PNEC. La même démarche a été adoptée pour traiter les données de toxicité chronique (Tab. 3). Si le jeu de données écotoxicologiques est suffisamment important, la méthode d'extrapolation statistique, évoquée précedemment, reste valable pour l'évaluation des effets en milieu marin.

Les sédiments représentent un compartiment important pour les écosystèmes aquatiques. De nombreux organismes effectuent une partie de leur cycle de vie au contact de cette matrice. Les substances les plus hydrophobes peuvent s'y fixer et s'y accumuler et donc présenter un risque pour les organismes benthiques. Pour les substances chimiques pour lesquelles des résultats d'essais sont disponibles sur des organismes benthiques, des PNEC peuvent également être estimées pour le sédiment en utilisant des facteurs d'extrapolation applicables aux valeurs écotoxicologiques les plus sensibles (Tab. 4).
Les résultats des bio-essais doivent être considérés avec précaution, ils sont en effet très variables selon le type de sédiment choisi. La granulométrie et la teneur en carbone organique agissent en effet sur la bio-disponibilité d'une substance chimique. De plus, les tests monospécifiques négligent les interactions possibles entre la faune sédimentaire et le comportement à long terme des polluants - interactions causées par la bioturbation et non estimables actuellement.

Dans le cas où il n'existe pas de données expérimentales vis-à-vis des organismes benthiques, la PNEC ne peut pas être déterminée par la méthode des facteurs d'extrapolation. La méthode du coefficient de partage eau-solide pour les sédiments est alors appliquée. Celle-ci présuppose que la sensibilité des espèces benthiques est identique à la sensibilité des espèces de la colonne d'eau et qu'il existe un équilibre entre la concentration en polluant dans l'eau interstitielle et la concentration dans le sédiment.

Les coefficients de partage entre les sédiments et l'eau peuvent être mesurés ou estimés à partir des caractéristiques du milieu et de la substance.

$$
\begin{gathered}
\text { PNEC }_{\text {sédiment marin }}=\mathrm{K}_{\mathrm{MES} / \mathrm{eau}} / \mathrm{RHO}_{\mathrm{MES}} \\
\times \mathrm{PNEC}_{\text {eau marine }} \times 1000
\end{gathered}
$$

où PNEC $_{\text {sédiment marin est la concen- }}$ tration sans effet prévisible dans le sédiment marin $(\mathrm{mg} / \mathrm{L}) ; \mathbf{K}_{\text {MES/eau }}$ est le coefficient de partage matière en suspension/eau $\left(\mathrm{m}^{3} / \mathrm{m}^{3}\right)$; RHO MES $_{\text {est }}$ la densité de la matière en suspension $\left(\mathrm{kg} / \mathrm{m}^{3}\right)$; et PNEC $_{\text {eau marine est }}$ la concentration sans effet prévisible dans l'eau de mer (mg/L). 
Tableau 3. Valeurs des facteurs d'extrapolation selon la nature et la qualité du jeu de données disponible pour calculer la PNEC aquatique.

Table 3. Assessment factors proposed for deriving sea water PNEC in relation to the characteristics of the available data sets.

\begin{tabular}{|c|c|}
\hline & $\begin{array}{c}\text { PNEC } \\
\text { eau marine }\end{array}$ \\
\hline $\begin{array}{l}\text { La plus faible } \mathrm{L}(\mathrm{E}) \mathrm{C} 50 \text { d'un essai court terme sur des représentants d'eau } \\
\text { douce ou salée de trois groupes taxonomiques (algues, crustacés et pois- } \\
\text { sons) de trois niveaux trophiques. }\end{array}$ & $10000^{(a)}$ \\
\hline $\begin{array}{l}\text { La plus faible } L(E) C 50 \text { d'un essai court terme sur des représentants d'eau } \\
\text { douce ou salée de trois groupes taxonomiques (algues, crustacés et pois- } \\
\text { sons) de trois niveaux trophiques, }+2 \text { groupes taxonomiques marins supplé- } \\
\text { mentaires (Echinodermes, Mollusques) }\end{array}$ & 1000 \\
\hline $\begin{array}{l}\text { Une NOEC (CSEO) chronique (test de reproduction d'un organisme } \\
\text { représentatif des eaux salines, ou des études sur la croissance sur poissons) }\end{array}$ & $1000^{(\mathrm{b})}$ \\
\hline $\begin{array}{l}\text { Deux NOEC chroniques pour au moins trois espèces représentant deux } \\
\text { niveaux trophiques (poissons et/ou daphnies et/ou algues) }\end{array}$ & $500^{(c)}$ \\
\hline $\begin{array}{l}\text { La plus faible NOEC chronique pour au moins trois espèces représentant } \\
\text { trois niveaux trophiques (normalement poissons et daphnies et algues) }\end{array}$ & $100^{(\mathrm{d})}$ \\
\hline $\begin{array}{l}\text { Deux NOEC chroniques pour au moins trois espèces représentant deux } \\
\text { niveaux trophiques (poissons et/ou daphnies et/ou algues) } \\
+1 \text { NOEC chronique supplémentaire sur un groupe taxonomique marin } \\
\text { (Echinodermes, Mollusques) }\end{array}$ & 50 \\
\hline $\begin{array}{l}\text { La plus faible NOEC chronique pour au moins trois espèces représen- } \\
\text { tant trois niveaux trophiques (normalement poissons et/ou daphnies et/ou } \\
\text { algues) } \\
+2 \text { NOEC chronique supplémentaire sur un groupe taxonomique marin } \\
\text { (Echinodermes, Mollusques) }\end{array}$ & 10 \\
\hline $\begin{array}{l}\text { Autre cas, y compris les données obtenues sur le terrain ou écosystèmes- } \\
\text { modèles, qui permettent d'appliquer des facteurs de sécurité plus précis }\end{array}$ & $\begin{array}{l}\text { Évaluation au } \\
\text { cas par cas }\end{array}$ \\
\hline
\end{tabular}

(a) L'utilisation d'un facteur d'extrapolation de 10000 permet de protéger l'écosystème de l'ensemble des variations et incertitudes répertoriées ci-dessus, en considérant qu'elles contribuent toues de façon significative à l'incertitude totale. Pour certaines substances, il est possible qu'une des composantes de l'incertitude soit plus importante ou négligeable par rapport aux autres. Dans ce cas on peut faire varier le facteur en fonction de ces données.

(b) Un facteur de 1000 s'applique à la NOEC générée par un essai à long terme (Crustacé ou poisson d'eau douce ou salée). II faut que la NOEC corresponde au niveau trophique ayant la CL 50 la plus basse dans les essais court terme. Dans le cas contraire, on ne peut considérer la NOEC comme protectrice d'autres espèces plus sensibles. On utilise alors la CL 50 la plus faible avec un facteur d'incertitude de 10000 pour déterminer la PNEC aquatique, sauf si cette PNEC est plus élevée que la PNEC calculée d'après la NOEC.

Un facteur d'extrapolation de 1000 s'applique aussi à la plus faible des NOECs couvrant deux niveaux trophiques (algues et/ou crustacé et/ou poisson d'eau douce ou salée) quand les NOECs n'ont pas été obtenues à partir des essais courts termes ayant la plus faible CL 50.

(c) Un facteur 500 s'applique à la plus basse des deux NOECs si celle-ci couvre le niveau trophique possédant la plus basse CL 50 dans les essais court terme. 
Tableau 3. Suite.

Table 3. Continued.

S'il est possible de déterminer que le groupe trophique non testé sera moins sensible que les deux groupes testés, alors un facteur d'incertitude de 100 peut être appliqué à la plus faible NOEC de seulement deux espèces.

Si des essais court terme sur espèces marines sont connus et indiquent que ces espèces ne sont pas les plus sensibles et que les NOECs générées par ces espèces ne seront pas plus faibles que celles déjà obtenues, un facteur d'incertitude réduit (à 100 si on ne dispose que d'un essai court terme, à 50 si deux essais court terme sur espèces marines sont disponibles) peut être appliqué à la plus faible NOEC de seulement deux espèces. Ceci est particulièrement important si la substance n'a pas le potentiel de se bio-accumuler.

(d) Un facteur de 100 s'applique lorsque des NOECs issues d'essais long terme sont disponibles pour au moins trois espèces d'eau douce ou salée (Algues, Crustacés et Poissons) de trois niveaux trophiques différents. La NOEC la plus basse sert à dériver la PNEC aquatique.

Le facteur d'extrapolation peut être réduit à 10 si :

- des tests court terme sur des espèces marines (Echinodermes, Mollusques) ont été réalisés et indiquent que ces espèces ne seront pas plus basses, que celles déjà obtenues.

- des tests court terme sur espèces marines ont indiqué qu'une de ces espèces est plus sensible et qu'un test long terme a été réalisé pour l'une d'elles. Ceci ne s'appliquera que si les NOECs générées ne sont pas plus faibles que celles déjà disponibles.

Tableau 4. Facteurs d'extrapolation pour l'estimation de la PNEC des sédiments marins.

Table 4. Assessment factors for derivation of marine sediment PNEC.

\begin{tabular}{|c|c|c|}
\hline \multicolumn{2}{|c|}{ Tests disponibles } & $\begin{array}{l}\text { Facteurs } \\
\text { d'extrapolation }\end{array}$ \\
\hline \multirow{2}{*}{$\begin{array}{l}\text { Toxicité } \\
\text { aiguë }\left(\mathrm{CL}_{50}\right)\end{array}$} & 1 test en eau douce ou marine & 10000 \\
\hline & $\begin{array}{l}2 \text { tests dont } 1 \text { au minimum pratiqué sur un } \\
\text { organisme marin d'un taxon polluo-sensible }\end{array}$ & 1000 \\
\hline \multirow{5}{*}{$\begin{array}{l}\text { Toxicité } \\
\text { chronique } \\
\left(\text { NOEC/CE }_{10}\right)\end{array}$} & 1 test en eau douce & 1000 \\
\hline & $\begin{array}{l}2 \text { tests en eau douce avec des espèces à } \\
\text { mode de vie et régime alimentaire différent }\end{array}$ & 500 \\
\hline & $\begin{array}{l}1 \text { test avec des espèces à mode de vie et } \\
\text { régime alimentaire différent }\end{array}$ & 100 \\
\hline & $\begin{array}{l}3 \text { tests avec des espèces à mode de vie et } \\
\text { régime alimentaire différent }\end{array}$ & 50 \\
\hline & $\begin{array}{l}3 \text { tests avec des espèces à mode de vie et } \\
\text { régime alimentaire différent, dont } 2 \text { au } \\
\text { minimum pratiqués sur des espèces marines }\end{array}$ & 10 \\
\hline
\end{tabular}




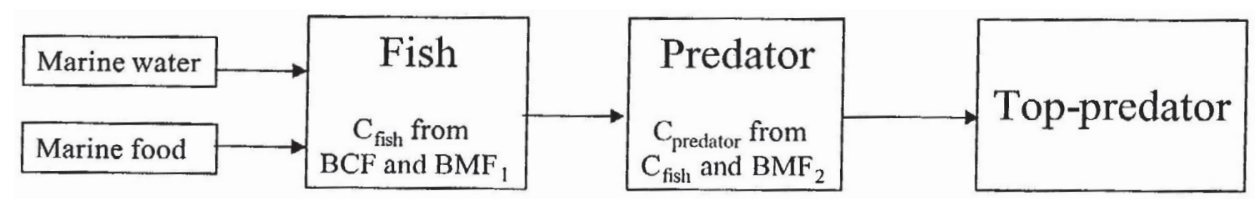

Fig. 3. Schéma simplifié de chaîne alimentaire pour évaluer l'empoisonnement secondaire.

Fig. 3. Secondary poisoning food chain.

Cependant, cette approche ne privilégie qu'une seule voie d'exposition, l'eau, au détriment d'autres comme l'ingestion de particules sédimentaires ou le contact cutané avec le sédiment. Or, ces dernières peuvent s'avérer importantes pour les espèces benthiques exposées à des substances lipophiles (log Kow > $3^{(2)}$ ), lesquelles franchissent aisément les membranes cellulaires. La méthode a donc été modifiée en appliquant un facteur de précaution supplémentaire de 10 pour les substances fortement adsorbables ( $\log$ Kow > 5), afin de prendre en compte l'ingestion des sédiments et le poids moléculaire de la substance, ce qui signifie concrètement que la valeur d'exposition (PEC) est multipliée par un facteur 10.

\section{6 Évaluation de l'intoxication secondaire chez les prédateurs marins}

L'impact sur la faune sauvage (oiseaux, mammifères) via le transfert des contaminants le long de la chaîne alimentaire définit ce que l'on appelle l'empoisonnement secondaire. II est évalué selon la même approche PEC/PNEC. Le modèle simplifié de chaîne alimentaire adopté est à trois niveaux : poisson, premier prédateur

(2) Kow : Coefficient de partage octanol/eau. et prédateur supérieur (Fig. 3). Trois facteurs de transfert trophique sont établis, le facteur de bioconcentration (BCF) et deux facteurs de biomagnification successifs (BMF1, BMF2), dont les valeurs génériques sont déterminées en fonction du coefficient de partage octanol/eau (Kow) de la substance et/ou des valeurs de BCF chez le poisson.

L'impact sur la faune sauvage (oiseaux, mammifères), via le transfert des contaminants le long de la chaîne alimentaire définit l'empoisonnement secondaire. Les valeurs des PNEC pour les prédateurs sont extrapolées à partir de données de toxicité par voie orale sur des mammifères ou des oiseaux terrestres, étant donné que dans la plupart des cas les données pour des espèces représentatives du milieu marin existent en nombre très limité. L'utilisation de données expérimentales d'espèces animales continentales pour la faune sauvage supérieure marine suppose qu'il existe une certaine corrélation interspécifique entre poulets-oiseaux marins et ratsmammifères marins.

\subsection{Cas des substances dangereuses}

Plusieurs considérations ont amené à proposer une approche spécifique 
Tableau 5. Critères pour définir une substance PBT (ECB, 2002).

Table 5. Criteria for identification of PBT substances.

\begin{tabular}{|c|c|}
\hline Critère & Substance PBT \\
\hline $\mathbf{P}$ & $\begin{aligned} \text { Demi-vie } & >60 \text { jours dans l'eau de mer } \\
& >180 \text { jours dans les sédiments marins }\end{aligned}$ \\
\hline$B$ & $\begin{array}{l}\text { BCF }>2000 \text { ou } \\
\text { LogKow }>4,5\end{array}$ \\
\hline$T$ & $\begin{array}{l}\text { Effets aigus : } \\
\mathrm{CL}(\mathrm{E}) 50<0,1 \mathrm{mg} / \mathrm{l} \text { pour les organismes aquatiques ou } \\
\mathrm{DL} 50<200 \mathrm{mg} / \mathrm{kg} / \mathrm{j} \text { (ingestion) } \\
\text { Effets chroniques } \\
\text { NOEC }<0,01 \mathrm{mg} / \mathrm{l} \text { pour les organismes aquatiques ou } \\
\text { NOEC }<30 \mathrm{mg} / \mathrm{kg} \text { d'aliment } \\
\text { Subtances reconnues cancérigène, mutagènes, toxiques pour } \\
\text { la reproduction ou provoquant des dysfonctionnements endo- } \\
\text { criniens }\end{array}$ \\
\hline
\end{tabular}

pour les substances dangereuses, basée non pas sur une évaluation du risque telle qu'elle est proposée classiquement dans le TGD, mais sur une évaluation du danger de ces substances selon des critères de persistance $(P)$, de bioaccumulation $(B)$ et de toxicité $(T)$. Cette approche a été estimée recevable compte tenu que le devenir ultime de la plupart des contaminants chimiques est le milieu marin, leurs effets à long terme ne sont pas prévisibles, par ailleurs une telle accumulation dans le milieu marin est difficilement réversible. II existe également une volonté de protéger les zones éloignées de l'océan mondial (environnement pristine) des substances dangereuses issues des activités humaines. La stratégie adoptée consiste, d'une part à définir les substances PBT selon un choix de critères définis dans le document, en accord avec ceux définis dans d'autres forums internationaux (ex. substances POP), d'autre part à évaluer les sources d'émission et les voies de transfert vers l'environnement marin afin de définir les mesures les plus appropriées pour réduire les apports. Le tableau 5 définit les critères PBT adoptés.

\section{EXEMPLE D'APPLICATION POUR LES REJETS CHIMIQUES DES INSTALLATIONS INDUSTRIELLES DU NORD-COTENTIN}

Dans le cadre de l'étude de l'impact des rejets chimiques des installations nucléaires du Nord-Cotentin sur l'environnement et la santé humaine (GRNC, 2002), la méthodologie d'évaluation des risques telle que décrite ci-dessus a été utilisée. Les compartiments environnementaux concernés sont les eaux marines (colonne d'eau, sédiments et prédateurs), les eaux douces (colonne d'eau et sédiments) et le sol.

Un total de 41 substances, parmi lesquelles une majorité de métaux et quelques organiques, est rejeté par les 
industries dans l'environnement. Dans l'eau de mer, l'évaluation des risques de 27 substances dites «toxiques» par rapport aux substances eutrophisantes (nitrates, phosphates...) et aux constituants majeurs de l'eau de mer $(\mathrm{Ca}, \mathrm{Cl} . .$.$) , a été réalisée. Par consé-$ quent, une concentration sans effet pour les écosystèmes marins a été proposée pour ces 27 substances. Trois exemples de substances (hydrazine, cobalt et chrome VI) pour lesquelles une $\mathrm{PNEC}_{\text {eau marine }}$ a été déterminée sont décrits ci-dessous afin d'illustrer différents cas de figure rencontrés lors de l'évaluation des effets et les méthodes utilisées. Seul un résumé est proposé ici, l'ensemble des données disponibles pour chaque substance est présenté en Annexe.

Dans le cas de l'hydrazine, des données écotoxicologiques sont disponibles sur des organismes marins et d'eau douce. Des données aiguës sur les trois niveaux trophiques de base (algues, crustacés, poissons) sont disponibles mais seulement des données long terme sur les algues sont disponibles. Cependant, les données d'écotoxicité aiguës relevées pour les algues sont nettement plus faibles que celles des autres niveaux trophiques. L'hydrazine agit donc spécifiquement sur les algues. Par conséquent, un facteur d'extrapolation de 100 a été appliqué à la plus faible NOEC observée pour l'algue marine Dunaliella tertiolecta, NOEC (6j) = 0,5 $\mu \mathrm{g} / \mathrm{l}$ plutôt qu'un facteur de 1000 du fait de la spécificité d'action de cette substance. La PNEC $_{\text {eau marine }}$ proposée pour l'hydrazine est donc de 0,005 $\mu \mathrm{g} / \mathrm{L}$.

Dans le cas du cobalt, la méthode des facteurs d'extrapolation a également été appliquée car les données n'étaient pas suffisantes pour déterminer une concentration par extrapolation statistique. Des données long terme sont disponibles pour les organismes d'eau douce et marins. Parmi les données long terme pour le milieu marin, seuls deux niveaux trophiques sont représentés (crustacés et poissons) en plus des données chroniques long terme disponibles chez les organismes d'eau douce pour trois niveaux trophiques. Par conséquent, selon le TGD un facteur d'extrapolation de 50 s'applique à la plus faible des NOECs qui est celle reportée pour Daphnia magna, NOEC $(21 \mathrm{j})=5 \mu \mathrm{g} / \mathrm{l}$.

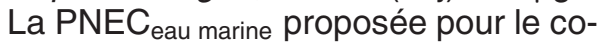
balt est donc de 0,1 $\mu \mathrm{g} / \mathrm{l}$.

Dans le cas du chrome VI, les données long terme à notre disposition sont nombreuses. Par conséquent, il a été possible d'appliquer la méthode d'extrapolation statistique. Cependant, par manque de données chroniques sur un niveau trophique important, les poissons, les jeux de données eau douce et eau marine confondus ont été utilisés. La courbe de distribution des données est présentée sur la figure 4.

La HC5 obtenue est de 7,8 $\mu \mathrm{g} / \mathrm{l}$ (IC_90 \% $=[3,4 ; 15,2]$. Des données long terme sur les poissons marins mais aussi sur d'autres niveaux trophiques importants comme les mollusques font défaut. De plus, le résultat du test vis-à-vis de Ceriodaphnia dubia est inférieur à la HC5. Or cette valeur est la moyenne géométrique de 18 résultats d'essai lors d'un essai circulaire, elle ne peut en conséquence être ignorée. Par conséquent, pour la détermination de la PNEC eau marine, $_{\text {, }}$ la borne inférieure de l'intervalle de confiance sur la HC5 est utilisée.

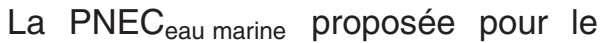


Toxicité long terme du chrome VI vis-à-vis des organismes

aquatiques

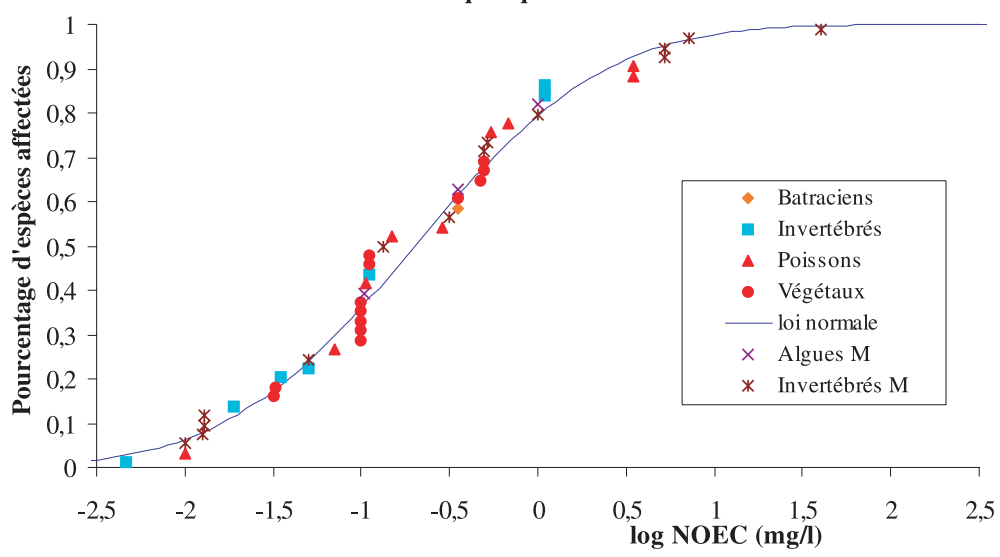

Fig. 4. Distribution de la toxicité chronique du chrome VI vis-à-vis des organismes aquatiques (eau douce et marine confondues).

Fig. 4. Distribution of chronic toxicity data for chromium VI on aquatic organisms (fresh water and marine species).

chrome $\mathrm{VI}$ est par conséquent de 3,4 $\mu \mathrm{g} / \mathrm{l}$.

Ce travail sur un grand nombre de substances a permis de mettre en évidence les différentes limites rencontrées lors de l'évaluation des effets pour le milieu marin. En effet, pour 21 sur les 27 substances rejetées, la PNEC a été déterminée à partir de données de toxicité long terme sur organismes d'eau douce et donc par l'application d'un facteur 10 supplémentaire sur la PNEC déterminée pour l'eau douce. Ce constat met en évidence le vrai manque de données d'écotoxicité sur organismes marins. Ce manque de données peut conduire à des incohérences et des valeurs peu significatives pour le milieu. C'est le cas, par exemple, du chrome III, pour lequel il n'a pas été possible de déterminer une PNEC pour le milieu marin. Pour cette substance, seules des don- nées long terme sur des organismes d'eau douce sont disponibles et l'application d'un facteur 10 supplémentaire sur la $\mathrm{PNEC}_{\text {eau douce }}$ conduit à une $P N E C_{\text {eau marine }}$ plus faible que celle déterminée pour le chrome VI reconnu comme plus toxique et pour lequel les données chroniques sont nombreuses.

Par ailleurs, il semble que lorsque des données chroniques sont disponibles en grand nombre pour les organismes marins et d'eau douce (cas de quelques métaux), les concentrations sans effet obtenues, d'une part en eau douce, d'autre part en eau marine sont proches. Ce constat ne remet pas pour autant en question le facteur 10 de différence proposé car la représentativité des espèces testées par rapport au milieu est faible. Ce sont bien souvent toujours les mêmes espèces qui sont testées aussi bien en eau douce qu'en eau marine. 
De plus, du fait du manque de représentativité des espèces marines dans les jeux de données et de l'absence de certains niveaux trophiques fondamentaux, la plupart des PNECs déterminées à partir de la méthode d'extrapolation statistique ont été obtenues à partir des jeux de données écotoxicologiques eau marine et eau douce confondus.

Cette étude a permis de travailler sur un grand nombre de substances et d'utiliser la méthode de détermination des effets à partir de jeux de données très différents. Pour le milieu marin, le manque de données de toxicité à long terme est clairement mis en évidence et par conséquent la nécessité de réaliser des tests sur des organismes marins afin d'affiner les concentrations sans effet prévisibles est nécessaire.

\section{CONCLUSION}

L'évaluation du risque chimique est reconnue comme un outil d'aide aux gestionnaires pour les aider à fixer les priorités et à développer des programmes de gestion environnementale. Toutefois, un certain nombre d'incertitudes existe dans cette procédure lorsqu'elle est appliquée aux milieux aquatiques:

- l'évaluation des dangers inhérents aux contaminants chimiques dans un environnement ne peut être connue que si ceux-ci sont identifiés, ce qui pose le problème du " criblage » des contaminants dans un environnement soumis à une forte pression anthropique ;

- la détermination de l'exposition des contaminants dans l'environnement dépend bien évidemment de la qualité des données disponibles, tributaires des méthodologies analytiques utilisées pour ce qui concerne les valeurs mesurées, mais également d'une connaissance des sources d'apports des contaminants, collectés et diffus, pour ce qui concerne les données calculées à l'aide de modèles ;

- l'évaluation des effets par substance présuppose des effets additifs alors que les effets synergiques ou antagonistes liés à une exposition de nombreuses substances ne peuvent être ignorés; par ailleurs d'autres effets cumulatifs sont également possibles avec d'autres agents de stress (variabilité naturelle de l'écosystème, surpêche, érosion, ... ).

L'un des enjeux majeurs en matière de gestion environnementale est la mise en œuvre de la Directive Cadre sur l'Eau (2000/60/CE) qui concerne tous les milieux aquatiques, incluant de ce fait les eaux marines côtières et les estuaires. L'objectif demandé aux États membres est de parvenir à un "bon état » des eaux d'ici 2015. Un second objectif concerne la suppression des substances toxiques, c'est-à-dire la réduction de la pollution due aux substances prioritaires et la suppression des émissions, rejets et pertes des substances dangereuses prioritaires. À cet effet, une liste de 33 substances prioritaires a été établie par les instances européennes (CE, 2001). Le « bon état » des eaux est défini selon deux approches complémentaires, l'une relative à l'état écologique basée sur une appréciation de la structure et du fonctionnement des écosystèmes aquatiques, la 
seconde relative au respect de normes de qualité environnementale physicochimique. Pour les contaminants chimiques, ces normes de qualité environnementales (NQE) sont définies sur la base des critères écotoxicologiques qui figurent dans le guide d'évaluation des risques (TGD).

En conclusion, le guide méthodologique européen (TGD) adapté au milieu marin devient, pour partie ou en totalité, un outil commun à trois dispositions, juridiques actuelles : la politique européenne sur les substances chimiques (évaluation des risques des substances chimiques), la Convention OSPAR (stratégie sur les substances dangereuses) et de la Directive Cadre sur l'Eau (définition des normes de qualité environnementale), ce qui souligne toute son importance.

\section{RÉFÉRENCES \\ BIBLIOGRAPHIQUES}

Aldenberg T. \& Slob W. (1993) Confidence limits for hazardous concentrations based on logistically distributed NOEC toxicity data. Ecotoxicology and Environmental Safety, 25 : 48-63.

Aldenberg T. \& Jaworska J.S. (2000) Uncertainty of the hazardous concentration and fraction affected for normal species sensitivity distributions. Ecotoxicology and Environmental Safety, 46(1) : 1-18

CE (1996) Technical Guidance Document in support of Commission Directive 93/67/EEC on risk assessment for new notified substances and commission Regulation (EC) $\mathrm{N}^{\circ} 1488 / 94$ on risk assessment for existing substances. Office for official Publications of the European Communities. Luxembourg.

CE (2001) Décision №2455/2001/CE du Parlement Européen et du Conseil du 20 novembre 2001 établissant la liste des substances prioritaires dans le domaine de l'eau et modifiant la directive 2000/60/CE. Journal officiel des Communautés européennes, 15/12/2001 : L331/1-L331/5.

ECB (2002) Revision of the Technical Guidance Document. Chap.4 Environmental risk assessment marine. European Chemicals Bureau, http://ecb.jrc.it

ECETOC (2001) Risk assessment in marine environments. Technical Report, No 82. European Centre for Ecotoxicology and Toxicology of Chemicals, Bruxelles : $135 \mathrm{pp}$.

GRNC (2002) Evaluation de l'impact des rejets chimiques des installations nucléaires du Nord-Cotentin sur la santé humaine et les écosystèmes. Groupe Radioécologique Nord-Cotentin (document non publié).

Hutchinson T.H., Scholz N. \& Guhl W. (1998) Analysis of the ECETOC Aquatic Toxicity (EAT) database IV-comparative toxicity of chemical substances to freshwater versus saltwater organisms. Chemosphere $36: 143-153$.

Lewis R. (1997) Dispersion in estuaries and coastal waters. Ed. John Wiley \& Sons : $313 \mathrm{pp}$.

Mackay D., Paterson S. \& Shiu W.Y. (1992) Generic models for evaluating the regional fate of chemicals. Chemosphere 24 (6) : 695-717.

Matthiessen P., Thain J.E., Law R.J. \& Fileman T.W. (1993) Attempts to assess the environmental hazard posed by complex mixtures of organic chemicals in UK estuaries. Marine Pollution Bulletin, 26(2) : 90-95.

Thomas K.V., Fileman T.W., Readman J.W. \& Waldock M.J. (2001) Antifouling paint booster biocides in the UK coastal environment and potential risks of biological effects. Marine Pollution Bulletin, 42(3) : 677-688.

Xie W.H., Shiu W.Y. \& Mackay D. (1997) A review of the effects of salts on the solubility of organic compounds in seawater. Marine Environmental research, 44 : 429-444. 


\title{
Annexe 1
}

\author{
Nom de la substance : Hydrazine \\ Identification : $\mathrm{n}^{\circ}$ CAS : 302-01-2 $\mathrm{n}^{\circ}$ EINECS : 206-114-9 \\ Formule brute : $\mathrm{N}_{2} \mathrm{H}_{4}$
}

\section{DEVENIR DANS L'ENVIRONNEMENT}

\section{I.1 Bioaccumulation dans les organismes aquatiques}

II n'existe pas de données de bioaccumulation pour l'hydrazine. Compte tenu de la valeur du Kow, l'accumulation de l'hydrazine dans les organismes semble peu probable.

Une estimation du BCF pour les poissons peut être obtenue à l'aide des relations de type (Q)SAR (C.E., 1996). Nous proposons d'utiliser la même valeur pour les mollusques et les crustacés.

Par conséquent, nous préconisons l'utilisation des BCF suivants :

BCF poissons $=0,06$

BCF mollusques $=0,06$

BCF crustacés $=0,06$

Nous ne tiendrons donc pas compte de l'hydrazine pour l'empoisonnement secondaire.

\section{I.2 Paramètres d'évaluation de l'exposition}

\begin{tabular}{|l|c|c|}
\hline \multicolumn{1}{|c|}{ Paramètre } & Valeur & Référence \\
\hline Masse molaire $(\mathrm{g} / \mathrm{mol})$ & 32.05 & \\
\hline Solubilité dans l'eau $(\mathrm{mg} / \mathrm{l})$ à $20^{\circ} \mathrm{C}$ & miscible & \\
\hline Pression de vapeur $\left(\mathrm{hPa}\right.$ à $\left.20^{\circ} \mathrm{C}\right)$ & $13,9-21,3$ & $\mathrm{BUA}, 1998$ \\
\hline Coefficient de partage octanol eau log Kow & $-1,37$ à $-0,6$ & $\mathrm{BUA}, 1998$ \\
\hline $\begin{array}{l}\text { Coefficient de partage eau-solide dans les sédiments : } \\
\text { log Kp }\end{array}$ & non attribué $(\mathrm{l} / \mathrm{kg})$ & \\
\hline $\begin{array}{l}\text { Coefficient de partage eau-solide dans les matières en } \\
\text { suspension : log Kp }\end{array}$ & non attribué $(\mathrm{l} / \mathrm{kg})$ & \\
\hline $\begin{array}{l}\text { Coefficient de partage eau-solide dans le sol : log } \mathrm{Kp}_{\text {Sol }} \\
(\mathrm{l} / \mathrm{kg})\end{array}$ & non attribué & \\
\hline
\end{tabular}

L'hydrazine a un faible potentiel d'adsorption sur la matière organique (sol ou sédiment) compte tenu de la valeur du Kow.

\section{DONNÉES ÉCOTOXICOLOGIQUES}

La majorité des données écotoxicologiques rapportées provient du RIVM (1999) et de BUA (1998). 


\section{II.1 Paramètres d'écotoxicité vis-à-vis des organismes aquatiques}

\section{II.1.1 Écotoxicité aiguë}

a) Organismes d'eau douce

\begin{tabular}{|c|c|c|c|c|c|}
\hline Toxicité & Espèces & $\begin{array}{l}\text { Substance } \\
\text { testée }\end{array}$ & $\begin{array}{c}\text { Critère d'effet } \\
\text { d'effet }\end{array}$ & $\begin{array}{l}\begin{array}{l}\text { Valeur } \\
\text { (mg/l) }\end{array}\end{array}$ & Référence \\
\hline \multirow[t]{3}{*}{ Algues } & $\begin{array}{l}\text { Chlorella stigmatophora } \\
\text { Milieu oligotrophe } \\
\text { Milieu eutrophe }\end{array}$ & $\mathrm{N}_{2} \mathrm{H}_{5} \mathrm{OH}$ & $\mathrm{CE}_{50}(6 \mathrm{j})$ & $\begin{array}{l}0,010 \\
0,019\end{array}$ & Dixon et al., 1979; \\
\hline & \begin{tabular}{|l} 
Selenastrum \\
capricornutum \\
Milieu oligotrophe \\
Milieu eutrophe
\end{tabular} & $\mathrm{N}_{2} \mathrm{H}_{5} \mathrm{OH}$ & $\mathrm{CE}_{50}(8 \mathrm{j})$ & $\begin{array}{l}0,002 \\
0,006\end{array}$ & Scherfig et al., 1978 \\
\hline & Chlorella pyrenoidosa & & $\mathrm{CL}_{50}(48 \mathrm{~h})$ & 0,1 & Heck et al., 1963 \\
\hline \multirow[t]{5}{*}{ Crustacés } & Daphnia magna & $\mathrm{N}_{2} \mathrm{H}_{5} \mathrm{OH}$ & $\mathrm{CE}_{50}(24 \mathrm{~h})$ & 0,81 & $\begin{array}{l}\text { Bringmann et Kühn, } \\
1977\end{array}$ \\
\hline & Daphnia magna & $\mathrm{N}_{2} \mathrm{H}_{5} \mathrm{OH}$ & $\mathrm{CE}_{50}(24 \mathrm{~h})$ & 2,3 & $\begin{array}{l}\text { Bringmann et Kühn, } \\
1982\end{array}$ \\
\hline & Daphnia pulex & $\mathrm{N}_{2} \mathrm{H}_{5} \mathrm{OH}$ & $\mathrm{CE}_{50}(48 \mathrm{~h})$ & $\begin{array}{l}0,19 \\
0,16\end{array}$ & Velte, 1984 \\
\hline & Hyalella azteca & $\mathrm{N}_{2} \mathrm{H}_{4}$ & $\mathrm{CE}_{50}(48 \mathrm{~h})$ & 0,04 & Fisher et al., 1980 \\
\hline & Asellidae & $\mathrm{N}_{2} \mathrm{H}_{4}$ & $\mathrm{CE}_{50}(72 \mathrm{~h})$ & 1,3 & Fisher et al., 1980 \\
\hline \multirow[t]{16}{*}{ Poissons } & Lepomis macrochirus & & $\mathrm{CL}_{50}(96 \mathrm{~h})$ & 1,08 & Fisher et al., 1978 \\
\hline & Lepomis macrochirus & & $\mathrm{CL}_{50}(96 \mathrm{~h})$ & 1,0 & Hunt et al, 1981 \\
\hline & Pimephales promelas & $\mathrm{N}_{2} \mathrm{H}_{5} \mathrm{OH}$ & $\mathrm{CL}_{50}(96 \mathrm{~h})$ & 5,98 & Velte, 1984 \\
\hline & Pimephales promelas & & $\mathrm{CL}_{50}(96 \mathrm{~h})$ & 4,5 & Cowen et al., 1981 \\
\hline & Salmo gairdneri & & $\mathrm{CL}_{50}(76 \mathrm{~h})$ & 6 & Henderson et al., 1983 \\
\hline & Brachydanio rerio & $\mathrm{N}_{2} \mathrm{H}_{5} \mathrm{OH}$ & $\mathrm{CL}_{50}(24 \mathrm{~h})$ & 1,17 & Proteau et al., 1979 \\
\hline & Carassius auratus & $\mathrm{N}_{2} \mathrm{H}_{5} \mathrm{OH}$ & $\mathrm{CL}_{50}(24 \mathrm{~h})$ & 1,48 & Proteau et al., 1979 \\
\hline & Ictalurus punctatus & $\mathrm{N}_{2} \mathrm{H}_{4}$ & $\mathrm{CL}_{50}(96 \mathrm{~h})$ & 1 & Fisher et al., 1980 \\
\hline & $\begin{array}{l}\text { Notemigonus } \\
\text { crysoleucas }\end{array}$ & & $\mathrm{CL}_{50}(96 \mathrm{~h})$ & 1,12 & Fisher et al., 1980 \\
\hline & Poecilia reticulata & & $\mathrm{CL}_{50}(96 \mathrm{~h})$ & 0,61 & Slonim, 1977 \\
\hline & $\begin{array}{l}\text { Leuciscus idus } \\
\text { melanotus }\end{array}$ & & $\begin{array}{l}\mathrm{CL}_{50}(48 \mathrm{~h}) \\
\text { Système } \\
\text { ouvert }\end{array}$ & 0,75 & $\begin{array}{l}\text { Junkhe et Lüdemann, } \\
1978\end{array}$ \\
\hline & Rutilus rutilus & $\mathrm{N}_{2} \mathrm{H}_{5} \mathrm{OH}$ & $\mathrm{CL}_{50}(24 \mathrm{~h})$ & 0,85 & Proteau et al., 1979 \\
\hline & Lepomis cyanellus & & $\mathrm{CL}_{50}(48 \mathrm{~h})$ & 5,1 & Heck et al., 1963 \\
\hline & Micropterus salmoides & & $\mathrm{CL}_{50}(48 \mathrm{~h})$ & 3,6 & Heck et al., 1963 \\
\hline & Ictalurus punctatus & & $\mathrm{CL}_{50}(48 \mathrm{~h})$ & 1,6 & Heck et al., 1963 \\
\hline & Gasterosteus aculeatus & & $\mathrm{CL}_{50}(96 \mathrm{~h})$ & 3,4 & Harrah, 1978 \\
\hline
\end{tabular}

b) Organismes marins

\begin{tabular}{|l|l|c|c|c|l|}
\hline Organisme & Espèce & $\begin{array}{c}\text { Substance } \\
\text { testée }\end{array}$ & $\begin{array}{c}\text { Critère } \\
\text { d'effet }\end{array}$ & $\begin{array}{c}\text { Valeur } \\
(\mu \mathrm{g} / \mathrm{l})\end{array}$ & Référence \\
\hline Algues & Dunaliella tertiolecta & $\mathrm{N}_{2} \mathrm{H}_{5} \mathrm{OH}$ & $\mathrm{CE}_{50}(10 \mathrm{j})$ & & Scherfig et al., \\
& Milieu oligotrophe & & & 0,8 & 1978 \\
& Milieu eutrophe & & & 1,1 & \\
\hline
\end{tabular}




\section{II.1.2 Écotoxicité chronique}

a) Organismes d'eau douce

\begin{tabular}{|c|c|c|c|c|c|}
\hline Organismes & Espèce & $\begin{array}{c}\text { Substance } \\
\text { testée }\end{array}$ & $\begin{array}{l}\text { Critère } \\
\text { d'effet }\end{array}$ & $\begin{array}{l}\text { Valeur } \\
(\mu \mathrm{g} / \mathrm{l})\end{array}$ & Référence \\
\hline \multirow[t]{3}{*}{ Algues } & $\begin{array}{l}\text { Chlorella stigmatophora } \\
\text { Milieu oligotrophe } \\
\text { Milieu eutrophe }\end{array}$ & $\mathrm{N}_{2} \mathrm{H}_{5} \mathrm{OH}$ & $\operatorname{NOEC~(6j)}$ & $\begin{array}{c}5 \\
15\end{array}$ & Dixon et al., 1979 \\
\hline & $\begin{array}{l}\text { Scenedesmus } \\
\text { quadricauda }\end{array}$ & $\mathrm{N}_{2} \mathrm{H}_{5} \mathrm{OH}$ & $\operatorname{NOEC}(8 \mathrm{j})$ & 5 & Bringmann et Kühn, 1978 \\
\hline & \begin{tabular}{|l|} 
Selenastrum \\
capricornutum \\
Milieu oligotrophe \\
Milieu eutrophe
\end{tabular} & $\mathrm{N}_{2} \mathrm{H}_{5} \mathrm{OH}$ & $\begin{array}{c}\text { NOEC (6 j) } \\
\text { 1 } \\
5\end{array}$ & & Scherfig et al.,1978 \\
\hline
\end{tabular}

b) Organismes marins

\begin{tabular}{|l|l|c|c|c|l|}
\hline Organismes & Espèce & $\begin{array}{c}\text { Substance } \\
\text { testée }\end{array}$ & $\begin{array}{c}\text { Critère } \\
\text { d'effet }\end{array}$ & $\begin{array}{c}\text { Valeur } \\
(\mu \mathrm{g} / \mathrm{l})\end{array}$ & Référence \\
\hline Algues & Dunaliella tertiolecta & $\mathrm{N}_{2} \mathrm{H}_{5} \mathrm{OH}$ & NOEC(6 j) & & Scherfig et al., 1978 \\
& Milieu oligotrophe & & 0,5 & \\
& Milieu eutrophe & & & 1,01 & \\
\hline
\end{tabular}

\section{II.2 Paramètres d'écotoxicité vis-à-vis des organismes du sédiment}

II n'est pas nécessaire de déterminer une PNEC pour le milieu sédimentaire du fait du faible potentiel d'adsorption de l'hydrazine sur les sédiments.

\section{II.3 Paramètres d'écotoxicité vis-à-vis des organismes terrestres}

\begin{tabular}{|l|l|c|c|c|}
\hline Organisme & Espèce & Critère d'effet & Valeur $(\mathbf{m g} / \mathbf{l})$ & Référence \\
\hline \multirow{4}{*}{ Plantes } & Cucurbita pepo & LOEC (48 h) & 10 & Heck et al., 1963 \\
\cline { 2 - 5 } & Arachis hypogaea & LOEC (48 h) & 100 & Heck et al., 1963 \\
\cline { 2 - 5 } & Zea mays & LOEC (48 h) & 1000 & Heck et al., 1963 \\
\hline
\end{tabular}

\section{II.4 Effets sur les prédateurs}

II n’existe pas de données de toxicité par voie orale sur les prédateurs.

\section{CONCENTRATIONS SANS EFFET PRÉVISIBLE POUR L'ENVIRONNEMENT (PNEC)}

Seules des données long terme sur les algues sont disponibles. Par ailleurs, les données d'écotoxicité aiguës relevées pour les algues sont nettement plus faibles que pour les autres niveaux trophiques. L'hydrazine agit spécifiquement 
sur les algues. Par conséquent, on appliquera un facteur d'incertitude de 100 à la plus faible NOEC déterminée par Scherfig et al (1978) pour Dunaliella tertiolecta, NOEC $(6 \mathrm{j})=0,5 \mu \mathrm{g} / \mathrm{l}$. Dunaliella tertiolecta étant une algue marine, la concentration sans effet prévisible pour l'environnement (PNEC) pour le milieu marin sera :

\section{PNECeau marine $=0,005 \mu \mathrm{g} / \mathrm{l}$.}

II n'est pas nécessaire de déterminer une PNEC pour l'eau douce puisque les rejets d'hydrazine se font en mer.

II n'est pas nécessaire de déterminer une PNEC pour le milieu terrestre car il n'existe pas de données d'exposition de ce compartiment.

Par manque de données de toxicité orale, il n'est pas possible de déterminer une PNEC pour les prédateurs.

\section{BIBLIOGRAPHIE}

Bringmann G. and Kühn R. (1977) - Results of toxic action of water pollutants on Daphnia magna (in german). Zeitschrift fuer Wasser- und Abwasser-Forschung, 10, 5, 161-166.

Bringmann G. and Kühn R. (1978) - Grenzwerte der Schadwirkung wassergefährdender stoffe gegen blaualgen (Microcystis aeruginosa) und grünalgen (Scenedesmus quadricauda) im zellvermehrungshemmtest. Vom Wasser, 50, 45-60.

Bringmann V.G.K., Kühn R. (1982) - Ergebnisse des Schadwirkung wassergefahrdender Stoffe gegen Daphnia magna in einem weiterentwickelten standardisierten Testverfahren. Z. Wasser Abwasser Forsch, 15, 1, 1-6.

BUA (1998) - Hydrazine, hydrazine hydrate and hydrazine sulfate. BUA Report 205 (December 1996).C.E. (1996) - Technical guidance document in support of Commission Directive 93/67/EEC on risk assessment for new notified substances and Commission Regulation (EC) No 1488/94 on risk assessment for existing substances. Office for Official Publications of the European Communities. Luxembourg.

C.E. (1996) - Technical guidance document in support of Commission Directive 93/67/EEC on risk assessment for new notified substances and Commission Regulation (EC) No $1488 / 94$ on risk assessment for existing substances. Office for Official Publications of the European Communities. Luxembourg.

Dixon P.S., Scherfig J. and Justice C.A. (1979) - Use of unicellular algae for evaluation of potential aquatic contaminants. US Air Force Aerospace Med. Res. Lab. AMRL-TR-7990.

Fisher J.W., Harrah C.B., Weaver L.K. and Wingo W.I. (1978) - Acute and behavioral effects of hydrazine on Lepomis macrochirus. Aerospace Med. Res. Lab. AMRL-TR-78-51.

Fisher J.W., Myers D.S. and Meyers M.L. (1980) - The effects of selected hydrazines upon fish and invertebrates. Air force Aerospace Med. Res. Lab. AMRL-TR-79-93.

Harrah C.B. (1978) Biological effects of aqueous hydrazine solutions. In : Proceedings of the conference of environmental chemistry : hydrazine fuels, Florida, Tyndall Air Force Base, Eds, 167-176.

Heck W.W., Bloodworth M.E., Clark W.J., Darling D.R. and Hoover W. (1963) Environmental pollution by missile propellants. Wright Patterson Air Force base, Aerospace Medical research laboratory. Ohio. AMRL-TDR-63-75. 
Henderson V., Fisher J.W., D'Alessandris R. and Livingstone J.M. (1983) - Effects of hydrazine on functional morphology of rainbow trout embryos and larvae. Trans. Am. Fish. Soc., 112, 100-104.

Hunt T.P., Fisher J.W., Livingstone J.M. and Putnam M.E. (1981) - Temperature effects on hydrazine toxicity to bluegills. Bull. Environm. Contam. Toxicol., 27, 588-595.

Juhnke J. and Lüdemann D. (1978) - Ergebnisse der Untersuchung von 200 chemischen verbindung auf akut : Fischtoxizität mit dem Goldorfentest. Zeitschrift Wasser-Abwasser Forsch., 11, 161-164.

Proteau J.P., Lim P. and Labat R. (1979) - Toxicité d'un dérivé bi-azolé, l'hydrate d'hydrazine, pour Carassius L., Rutilus rutilus L., et différents stades de développement de Brachydanio rerio H.B. Ann. Limnol., 15, 337-346.

RIVM (1999) - Environmental Risk Limits in the Netherlands. RIVM. 601640001.

Scherfig J., Dixon P.S. and C.A. Justice (1978) - Environmental quality research, use of unicellular algae for evaluation of potential aquatic contaminants. US Air Force Aerospace Med. Res. Lab. Third annual report. AMRL-TR-78-36.

Slonim A.R. (1977) - Acute toxicity of selected hydrazines to the common guppy. Water Research, 11, 889-895.

Velte J.S. (1984) - Acute toxicity of hydrazine hydrate to the fathead minnow (Pimephales promelas) and Daphnid (Daphnia pulex). Bull. Environ. Contam. Toxicol., 33, 598-604. 


\section{Annexe 2}

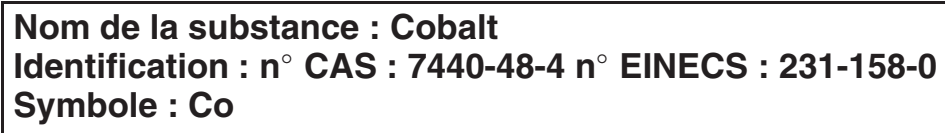

\section{DEVENIR DANS L'ENVIRONNEMENT}

\section{I.1 Bio-accumulation dans les organismes aquatiques}

Le G.R.N.C. (1999) s'est accordé pour utiliser les BCF suivants :

BCF poissons $=200$ avec un facteur correctif de 0,8 et 2 (avant et après 1990), soit un BCF de 160 et $\mathbf{4 0 0}$ (avant et après 1990).

BCF mollusques filtreurs (moules) $=2000$ avec un facteur correctif de 0,5 et 2 (avant et après 1990), soit un BCF de 1000 et $\mathbf{4 0 0 0}$ (avant et après 1990).

BCF mollusques non filtreurs (patelles) $=2000$ avec un facteur correctif de 0,5 et 2 (avant et après 1990), soit un BCF de 1000 et $\mathbf{4 0 0 0}$ (avant et après 1990). BCF crustacés $=5000$ avec un facteur correctif de 0,5 et 1,3 (avant et après 1990), soit un BCF de 2500 et $\mathbf{6 5 0 0}$ (avant et après 1990).

Par conséquent, pour l'empoisonnement secondaire nous utiliserons un BCF de 1000 avant 1990 et 4000 après 1990.

\section{I.2 Paramètres d'évaluation de l'exposition}

\begin{tabular}{|c|c|c|}
\hline Paramètres & Valeurs & Références \\
\hline Masse molaire $(\mathrm{g} / \mathrm{mol})$ & 58,933 & \\
\hline Pression de vapeur (hPa à $1050^{\circ} \mathrm{C}$ ) & $7.10^{-5}$ & IUCLID, 2000 \\
\hline Solubilité dans l'eau $(\mathrm{mg} / \mathrm{l})$ à $20^{\circ} \mathrm{C}$ & Non soluble & IUCLID, 2000 \\
\hline $\begin{array}{l}\text { Coefficient de partage eau-solide dans les sédiments: } \\
\text { log } \mathrm{Kp}_{\text {Sed }}(\mathrm{l} / \mathrm{kg})\end{array}$ & $\begin{array}{l}3,6 \\
3,2\end{array}$ & $\begin{array}{l}\text { RIVM,1999 } \\
\text { GRNC, } 1999\end{array}$ \\
\hline $\begin{array}{l}\text { Coefficient de partage eau-solide dans les matières en } \\
\text { suspension : log } \mathrm{Kp}_{M E S}(\mathrm{l} / \mathrm{kg})\end{array}$ & $\begin{array}{c}3,6 \\
4,7 \text { (médiane de } \\
29 \text { mesures) }\end{array}$ & $\begin{array}{l}\text { RIVM, } 1999 \\
\text { US-EPA, } 1999\end{array}$ \\
\hline Coefficient de partage eau-solide dans le sol : $\log \mathrm{Kp}_{\text {Sol }}(\mathrm{l} / \mathrm{kg})$ & $\begin{array}{c}1,6 \\
\text { Sable : } 1,78 \\
\text { Terreau : } 3,11 \\
\text { Argile : } 2,73 \\
\text { Organique : } 2,99 \\
2,1 \text { (médiane de } \\
11 \text { mesures) }\end{array}$ & $\begin{array}{l}\text { RIVM,1999 } \\
\text { IAEA, } 1994 \\
\text { US-EPA, } 1999\end{array}$ \\
\hline
\end{tabular}

Concernant le coefficient de partage eau-solide dans le sol, nous suggérons de prendre la valeur de 2,1 proposée par l'US-EPA (1999) et qui est intermédiaire entre la valeur proposée par le RIVM (1999) et celles proposées par l'IAEA (1994). 


\section{DONNÉES ÉCOTOXICOLOGIQUES}

\section{II.1 Paramètres d'écotoxicité vis-à-vis des organismes aquatiques}

\section{II.1.1 Écotoxicité aiguë}

a) Organismes d'eau douce

Étant donné le nombre élevé de données d'écotoxicité disponibles pour les organismes d'eau douce, seuls les essais chroniques sont retenus.

b) Organismes marins

\begin{tabular}{|l|l|c|c|c|l|}
\hline & Espèce & $\begin{array}{c}\text { Substance } \\
\text { testée }\end{array}$ & $\begin{array}{c}\text { Critère } \\
\text { d'effet }\end{array}$ & $\begin{array}{c}\text { Valeur } \\
\text { (mg Co/l) })\end{array}$ & Référence \\
\hline \multirow{2}{*}{ Algues } & Nitzschia closterium & $\mathrm{CoSO}_{4}$ & $\mathrm{CE}_{50}(96 \mathrm{~h})$ & 24 & Rosko et Rachlin, 1975 \\
\cline { 2 - 6 } & Ditylum brightwelli & $\mathrm{CoCl}_{2}$ & $\mathrm{CE}_{50}(5 \mathrm{j})$ & 0,3 & $\begin{array}{l}\text { Canterford et Canterford, } \\
1980\end{array}$ \\
\hline \multirow{2}{*}{ Crustacés } & Artemia salina & $\mathrm{Co}\left(\mathrm{NO}_{3}\right)_{3}$ & $\mathrm{CL}_{50}(48 \mathrm{~h})$ & 170 & Kissa et al., 1984 \\
\cline { 2 - 6 } & Artemia salina & $\mathrm{Co}\left(\mathrm{NO}_{3}\right)_{3}$ & $\mathrm{CE}_{50}(48 \mathrm{~h})$ & 10 & Kissa et al., 1984 \\
\cline { 2 - 6 } & Nitocra spinipes & $\mathrm{CoCl}_{2}$ & $\mathrm{CL}_{50}(96 \mathrm{~h})$ & 4,5 & Bengtsson, 1978 \\
\hline
\end{tabular}

\section{II.1.2 Écotoxicité chronique}

Les données sont pour la plupart extraites de RIVM (1999).

a) Organismes d'eau douce

\begin{tabular}{|c|c|c|c|c|c|}
\hline & Espèces & Substance & $\begin{array}{l}\text { Critère } \\
\text { d'effet }\end{array}$ & $\begin{array}{c}\text { Valeur } \\
(\mu \mathrm{g} \mathrm{Co} / \mathrm{l})\end{array}$ & Référence \\
\hline \multirow[t]{5}{*}{ Algues } & Spirulina platensis & $\mathrm{CoCl}_{2}$ & $\operatorname{NOEC}(7 \mathrm{j})$ & 500 & Sharma et al., 1987 \\
\hline & Chlorella pyrenoidosa & $\mathrm{CoSO}_{4}$ & NOEC (6 j) & 58 & Wong, 1980 \\
\hline & Chlorella pyrenoidosa & $\mathrm{CoSO}_{4}$ & $\operatorname{NOEC}(6 \mathrm{j})$ & 69 & Wong, 1980 \\
\hline & $\begin{array}{l}\text { Selenastrum } \\
\text { capricornutum }\end{array}$ & & $\operatorname{NOEC~(72~h)~}$ & $10-15$ & TNO, 1992 \\
\hline & Scenedesmus & $\mathrm{CoCl}_{2}$ & NOEC (96 h) & 500 & $\begin{array}{l}\text { Bringmann et Kühn, } \\
1959\end{array}$ \\
\hline Crustacés & Daphnia magna & $\mathrm{CoCl}_{2}$ & $\operatorname{NOEC}(21 \mathrm{j})$ & 5 & $\begin{array}{l}\text { Biesinger et Christensen, } \\
1972\end{array}$ \\
\hline \multirow[t]{3}{*}{ Poissons } & Pimephales promelas & & MATC (28 j) & 290 & Diamond et al., 1992 \\
\hline & Brachydanio rerio & $\mathrm{CoCl}_{2}$ & $\operatorname{NOEC}(16 \mathrm{j})$ & 60 & Dave et Xiu, 1991 \\
\hline & Cyprinus carpio (œufs) & $\mathrm{CoCl}_{2}$ & NOEC & 110 & Kapur et Yadav, 1982 \\
\hline
\end{tabular}

b) Organismes marins

\begin{tabular}{|l|l|l|l|c|l|}
\hline & Espèces & Substance & $\begin{array}{l}\text { Critère } \\
\text { d'effet }\end{array}$ & $\begin{array}{c}\text { Valeur } \\
(\mathbf{m g ~ C o / l})\end{array}$ & Référence \\
\hline \multirow{2}{*}{ Crustacés } & Carcinus maenas (adulte) & $\mathrm{CoCl}_{2}$ & NOEC $(10 \mathrm{j})$ & 110 & Amiard, 1976 \\
\cline { 2 - 6 } & Homarus vulgaris (larves stade 3) & $\mathrm{CoCl}_{2}$ & NOEC $(9 \mathrm{j})$ & 0,45 & Amiard, 1976 \\
\hline Poissons & Blennius pholis (adulte) & $\mathrm{CoCl}_{2}$ & NOEC $(15 \mathrm{j})$ & 45 & Amiard, 1976 \\
\hline
\end{tabular}




\section{II.2 Paramètres d'écotoxicité vis-à-vis des organismes du sédiment}

II n'existe pas de données valides sur organismes benthiques.

\section{II.3 Paramètres d'écotoxicité chronique sur les organismes terrestres}

\begin{tabular}{|l|l|l|c|c|l|}
\hline Organismes & Espèce & Substance & $\begin{array}{c}\text { Critère } \\
\text { d'effet }\end{array}$ & $\begin{array}{c}\text { Valeur } \\
\mathbf{( m g / k g})\end{array}$ & Référence \\
\hline Annélides & Eisenia fetida & $\mathrm{CoCl}_{2}$ & NOEC $(24 \mathrm{sem})$ & 240 & Neuhauser et al., 1984 \\
\cline { 2 - 6 } & Eisenia fetida & & NOEC $(8 \mathrm{sem})$ & $300-3000$ & Hartenstein et al., 1981 \\
\hline
\end{tabular}

\section{II.4 Effet sur les prédateurs}

II n'existe pas de données de toxicité par voie orale chez les mammifères.

\section{CONCENTRATIONS SANS EFFET PRÉVISIBLE POUR L'ENVIRONNEMENT (PNEC)}

Des données sont disponibles pour organismes marins et organismes d'eau douce. Une PNEC peut être déterminée pour chacun de ces compartiments.

Pour l'eau douce, des données d'écotoxicité chronique sont disponibles pour les trois niveaux trophiques (algues, crustacés et poissons). Un facteur d'incertitude de 10 est donc applicable à la plus faible valeur d'écotoxicité chronique. La NOEC la plus faible est celle reportée pour Daphnia magna par Biesinger and Christensen (1972) : NOEC (21j) $=5 \mu \mathrm{g} / \mathrm{l}$. D'où :

\section{PNECeau douce $=0,5 \mu \mathrm{g} / \mathrm{l}$.}

Pour le milieu marin, des données chroniques sur organismes marins sont disponibles pour deux niveaux trophiques (crustacés et poissons) en plus des données chroniques disponibles pour trois niveaux trophiques d'espèces d'eau douce. Un facteur d'incertitude de 50 s'applique donc à la plus faible des NOECs qui est celle reportée pour Daphnia magna par Biesinger and Christensen (1972) . D'où :

\section{PNECeau marine $=0,1 \mu \mathrm{g} / \mathrm{l}$.}

Compte tenu de l'absence de résultats de toxicité vis-à-vis des organismes benthiques, il n'est pas possible de dériver une PNEC pour les sédiments.

Il existe des résultats d'essais long terme sur des vers de terre (NOEC (24 sem) $=240 \mathrm{mg} / \mathrm{kg}$ ). La PNEC est obtenue à l'aide d'un facteur d'extrapolation de 100. D'où :

$$
\text { PNECsol = 2,4 } \mathrm{mg} / \mathrm{kg} \text { sol sec. }
$$

Compte tenu du manque de données, il n'est pas possible de déterminer une PNEC pour les prédateurs. 


\section{BIBLIOGRAPHIE}

Amiard J.C. (1976) - Etude expérimentale de la toxicité aiguë de sels de cobalt, d'antimoine, de strontium et d'argent chez quelques crustacés et leurs larves et chez quelques téléostéens. Rev. Intern. Océanogr. Med., 43, 79-95.

Bengtsson B.E. (1978) - Use of a harpacticoid copepod in toxicity tests. Mar Pollut Bull, 9 , 9, 238-241.

Biesinger K.E. and Christensen G.M. (1972) - Effects of various metals on the survival, growth, reproduction, and metabolism of Daphnia magna. J.Fish. Res. Board Can., 29, 1691-1700.

Bringmann G. and Kühn R. (1959) - Vergleichende wasser-toxikologische Untersuchungen an Bakterien, Algen und Kleinkrebsen. Gesundheit-Ingenieur., 80, 115-120.

Canterford G.S. and Canterford D.R. (1980) - Toxicity of Heavy Metals to the Marine Diatom Ditylum brightwellii (West) Grunow : Correlation between Toxicity and Metal Speciation. J. Mar. Biol. Assoc. U.K., 60, 1, 227-242.

C.E. (1996) - Technical guidance document in support of Commission Directive 93/67/EEC on risk assessment for new notified substances and Commission Regulation (EC) N ${ }^{\circ}$ $1488 / 94$ on risk assessment for existing substances. Office for Official Publications of the EC. Luxembourg.

Dave G. and Xiu R. (1991) - Toxicity of mercury, copper, nickel, lead and cobalt to embryos and larvae of zebrafish, Brachydanio rerio. Arch. Environ. Contam. Toxicol., 21, 126134.

Diamond J.M., Winchester E.L., Mackler D.G., Rasnake W.J., Fanelli J.K. and Gruber D. (1992) - Toxicity of cobalt to freshwater indicator species as a function of water hardness. Aquatic toxicology, 22, 3, 163-179.

G.R.N.C. (1999) - Modèles de transfert des radionucléides dans l'environnement. Groupe Radioécologie Nord Cotentin.

Hartenstein R., Neuhauser E.F. and Nakahara A. (1981) - Effects of heavy metal and other elemental additives to activated sludge on growth of Eisenia fetida. J.Environ.Qual., 10, 3, 372-376.

IAEA (1994) Handbook of parameter values for the prediction of radionuclide transfer in temperate environments. International Atomic Energy Agency, Vienna, 1994.

IUCLID (2000) International Uniform Chemical Information Database.

Kapur K. and Yadav N.A. (1982) - The effects of certain heavy metal salts on the development of eggs in common carp, Cyprinus carpio, var. communis. Acta Hydrochim. Hydrobiol., 10, 5, 517-522.

Kissa E., Moraitou-Apostolopoulou M. and Kiortsis V. (1984) - Effects of four heavy metals on survival and hatching rate of Artemia salina (L.). Arch. Hydrobiol., 100, 255-264.

Neuhauser E.F., Meyer J.A., Malecki M.R. and Thomas J.M. (1984) - Dietary cobalt supplements and the growth and reproduction of the earthworm Eisenia fetida. Soil. Biol. Biochem., 16, 521-525.

RIVM (1999) - Environmental Risk Limits in the Netherlands. RIVM. 601640001.

Rosko J.J. and Rachlin J.W. (1975) - The effect of copper, zinc, cobalt and manganese on the growth of the marine diatom Nitzschia closterium. Bull. of the Torrey Bot. Club, 102, 3, 100-106.

Sharma R.M., Panigrahi S. and Azeez P.A. (1987) - Effect of cobalt on the primary productivity of Spirulina platensis. Bull. Enviro. Contam. Toxicol., 39, 4, 716-720.

TNO (1992) - TNO. Cobalt Development Institute. IMW-R-92/277. 
US-EPA (1999) - Partition coefficients for metals in surface water, soil and waste. Draft of June 22, 1999. US-EPA, Office of solid waste, Washington DC.

Wong M.H. (1980) - Toxic effects of cobalt and zinc to Chlorella pyredoinosa in soft and hard water. Microbios, 28, 19-25. 


\section{Annexe 3}

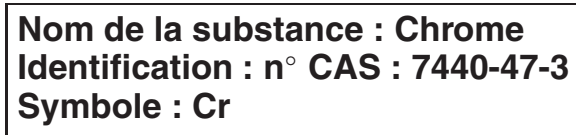

Le chrome est en cours d'évaluation dans le cadre du règlement (CEE) $n^{\circ}$ 793/93 du Conseil concernant l'évaluation et le contrôle des risques présentés par les substances existantes ${ }^{(3)}$. Nous pensons qu'il est préférable de s'appuyer sur les résultats de cette évaluation afin d'être homogène avec toute autre étude qui pourrait se faire au niveau européen. Par conséquent, les données présentées ci-après proviennent en grande partie du dernier projet de l'évaluation des risques du chrome (C.E., 2001).

\section{DEVENIR DANS L'ENVIRONNEMENT}

Le chrome existe sous plusieurs degrés d'oxydation, principalement chrome III et un peu chrome VI. Le chrome VI est largement transformé en chrome III dans les sols et les sédiments (favorisé en conditions anaérobiques et à un pH faible).

La solubilité du chrome VI est importante alors que le chrome III est généralement peu soluble.

Dans les sédiments et le sol, le chrome III s'adsorbe plus que le chrome VI. Dans l'eau, ils ont des coefficients de partage avec les matières en suspension similaires.

\section{I.1 Bio-accumulation dans les organismes aquatiques}

Le chrome VI ne s'accumule pas dans les poissons (des BCF pour le CrVI de l'ordre de 1 ont été rapportés par l'US-EPA (1980) et Calamari (1982)).

Le chrome III semble s'accumuler un peu plus que le chrome VI chez les poissons, que les organismes soient exposés à du chrome III ou à du chrome $\mathrm{VI}$ : des BCF de 260 à 800 (poids sec) ont été mesurés sur diverses espèces de poissons (Lepomis macrochirus, Alosa aestivalis, Notemigonus crysoleucas et Esox niger) par Giesy et Wiener (1977).

Dans des mollusques marins (Balanus sp.), des BCF de 543 (poids sec) et 380 (ce qui correspond à des BCF d'environs 110 et 80 respectivement en poids frais) ont été rapportés respectivement pour le chrome VI et Chrome III par Van Weerelt et al. (1984). Des BCF de 4,3 à 7,4 (poids sec) ont été mesurés pour la langoustine par Hernandez et al. (1986) après exposition au chrome VI (ce qui est équivalent à un BCF d'environ 2 en poids frais).

L'IAEA (1985) rapporte des BCF de 200 pour les poissons, 500 pour les crustacés et 800 pour les mollusques pour le chrome sans préciser sa forme chimique.

(3) JOCE Nº L84 du 5.4 .93 
Ces valeurs semblent plutôt correspondre aux valeurs rapportées par ailleurs pour le chrome III.

Par conséquent, nous préconisons l'utilisation des valeurs suivantes :

\section{Chrome VI}

BCF poissons $=1$

BCF mollusques $=110$

BCF crustacés $=2$

\section{Chrome III}

BCF poissons $=200$

BCF mollusques $=800$

BCF crustacés $=500$

\section{I.2 Paramètres d'évaluation de l'exposition}

\begin{tabular}{|c|c|c|c|}
\hline Paramètre & & Valeur & Référence \\
\hline Masse molaire ( $\mathrm{g} / \mathrm{mol})$ & & 52 & \\
\hline Pression de vapeur à $20^{\circ} \mathrm{C}(\mathrm{Pa})$ & & - & \\
\hline Solubilité à $20^{\circ} \mathrm{C}(\mathrm{g} / \mathrm{l})$ & $\begin{array}{l}\mathrm{Cr} \mathrm{O}_{3} \\
\mathrm{Na}_{2} \mathrm{Cr} \mathrm{O}_{4} \\
\mathrm{Na}_{2} \mathrm{Cr}_{2} \mathrm{O}_{7} \\
\mathrm{~K}_{2} \mathrm{Cr}_{2} \mathrm{O}_{7}\end{array}$ & $\begin{array}{c}625 \\
530 \\
2355 \\
115\end{array}$ & $\begin{array}{l}\text { HSDB, } 1999 ; \\
\text { C.E., } 2001\end{array}$ \\
\hline $\begin{array}{l}\text { Coefficient de partage eau-solide dans les sédiments: } \\
\text { log } \mathrm{Kp}_{\text {Sed }}(\mathrm{l} / \mathrm{kg})\end{array}$ & & 5,28 & RIVM, 1999 \\
\hline $\begin{array}{l}\text { Coefficient de partage eau-solide dans les matières } \\
\text { en suspension : log } \mathrm{Kp}_{M E S}(\mathrm{l} / \mathrm{kg})\end{array}$ & $\begin{array}{l}\mathrm{CrVI} \\
\mathrm{CrIII} \\
\mathrm{Cr}\end{array}$ & $\begin{array}{c}2,3 \\
5,5 \\
5,46\end{array}$ & $\begin{array}{l}\text { C.E., } 2001 \\
\text { C.E., } 2001 \\
\text { RIVM, } 1999\end{array}$ \\
\hline $\begin{array}{l}\text { Coefficient de partage eau-solide dans le sol : } \\
\log \mathrm{Kp}_{\text {Sol }}(\mathrm{l} / \mathrm{kg})\end{array}$ & & 2,04 & RIVM, 1999 \\
\hline
\end{tabular}

Les coefficients de partage eau-solide dans les matières en suspension proposés correspondent à ceux mesurés dans des conditions neutres ou basiques.

\section{DONNÉES ÉCOTOXICOLOGIQUES}

\section{II.1 Paramètres d'écotoxicité vis-à-vis des organismes aquatiques}

\section{II.1.1 Écotoxicité aiguë}

Étant donné le nombre élevé de données d'écotoxicité disponibles pour les algues, les micro-crustacés et les poissons pour le chrome III et le chrome VI, seuls les essais chroniques sont retenus. 


\section{II.1.2 Écotoxicité chronique}

\section{Chrome VI}

Les données présentées ici ont été sélectionnées et validées sur la base des paramètres toxicologiques, du milieu et des conditions d'essai. Ils sont identiques à ceux présentés dans C.E. (2001).

Lorsque plusieurs données existent sur une même espèce, la variabilité des résultats provient généralement des différences de conditions d'essai (différence de $\mathrm{pH}$, de dureté...).

Les LOEC sont fournies lorsque aucune concentration sans effet (hormis les témoins) n'a été testée.

\section{a) Organismes d'eau douce}

\begin{tabular}{|c|c|c|c|c|}
\hline Espèce & $\begin{array}{c}\text { substance } \\
\text { testée }\end{array}$ & Critère d'effet & $\begin{array}{c}\mathrm{Cr} \\
\mathrm{mg} / \mathrm{L}\end{array}$ & Référence \\
\hline \multicolumn{5}{|l|}{ Algues } \\
\hline Chlamydomonas sp & $\mathrm{K}_{2} \mathrm{Cr}_{2} \mathrm{O}_{7}$ & NOEC (c) $10 \mathrm{j}$ & 0,5 & Cairns Jr. et al., 1978 \\
\hline Chlorella vulgaris & $\mathrm{K}_{2} \mathrm{Cr}_{2} \mathrm{O}_{7}$ & $\mathrm{CE}_{50}(\mathrm{c}) 72 \mathrm{~h}$ & 0,47 & Jouany et al., 1982 \\
\hline Chlorella pyrenoidosa & $\mathrm{K}_{2} \mathrm{Cr}_{2} \mathrm{O}_{7}$ & NOEC (b) $96 \mathrm{~h}$ & 0,1 & Meisch et Schmitt-Beckmann, 1979 \\
\hline Chlorella sp. & $\mathrm{K}_{2} \mathrm{Cr}_{2} \mathrm{O}_{7}$ & NOEC (b) $96 \mathrm{~h}$ & 0,1 & Meisch et Schmitt-Beckmann, 1979 \\
\hline Lyngbya & $\mathrm{K}_{2} \mathrm{Cr}_{2} \mathrm{O}_{7}$ & NOEC (c) $18 \mathrm{j}$ & 0,1 & Cairns Jr. et al., 1978 \\
\hline Microcystis aeruginosa & $\mathrm{K}_{2} \mathrm{Cr}_{2} \mathrm{O}_{7}$ & $\operatorname{NOEC}(\mathrm{c}) 96 \mathrm{~h}$ & 0,35 & Sloof et Canton, 1983 \\
\hline $\begin{array}{l}\text { Scenedesmus } \\
\text { pannonicus }\end{array}$ & $\mathrm{K}_{2} \mathrm{Cr}_{2} \mathrm{O}_{7}$ & NOEC $96 \mathrm{~h}$ & 0,11 & Sloof et Canton, 1983 \\
\hline \multirow{2}{*}{$\begin{array}{l}\text { Scenedesmus } \\
\text { subspicatus }\end{array}$} & $\mathrm{K}_{2} \mathrm{Cr}_{2} \mathrm{O}_{7}$ & $\mathrm{CE}_{10}(\mathrm{c}) 72 \mathrm{~h}$ & 0,64 & Kuhn et Pattard, 1990 \\
\hline & & $\mathrm{CE}_{10}(\mathrm{~b}) 72 \mathrm{~h}$ & 0,032 & \\
\hline \multirow{3}{*}{$\begin{array}{l}\text { Selenastrum } \\
\text { capricornutum }\end{array}$} & $\mathrm{K}_{2} \mathrm{Cr}_{2} \mathrm{O}_{7}$ & $\mathrm{CE}_{10}(\mathrm{c}) 72 \mathrm{~h}$ & 0,11 & Nyholm, 1991 \\
\hline & $\mathrm{K}_{2} \mathrm{Cr}_{2} \mathrm{O}_{7}$ & $\mathrm{CE}_{10}(\mathrm{c}) 72 \mathrm{~h}$ & 0,01 & Christensen et al., 1983 \\
\hline & & & 0,033 & Moyenne géométrique (croissance) \\
\hline \multicolumn{5}{|l|}{ Plantes aquatiques } \\
\hline Lemna gibba & $\mathrm{Na}_{2} \mathrm{CrO}_{4}$ & NOEC (c) $8 \mathrm{j}$ & 0,1 & Staves et Knaus, 1985 \\
\hline Lemna minor & $\mathrm{K}_{2} \mathrm{Cr}_{2} \mathrm{O}_{7}$ & NOEC (c) $7 \mathrm{j}$ & 0,11 & Sloof et Canton, 1983 \\
\hline Spirodela polyrhiza & $\mathrm{Na}_{2} \mathrm{CrO}_{4}$ & NOEC (c) $8 \mathrm{j}$ & 0,1 & Staves et Knaus, 1985 \\
\hline Spirodela punctata & $\mathrm{Na}_{2} \mathrm{CrO}_{4}$ & NOEC (c) $8 \mathrm{j}$ & 0,5 & Staves et Knaus, 1985 \\
\hline \multicolumn{5}{|l|}{ Crustacés } \\
\hline Ceriodaphnia dubia & $\mathrm{K}_{2} \mathrm{Cr}_{2} \mathrm{O}_{7}$ & NOEC (r) $7 \mathrm{j}$ & 0,0047 & De Graeve et al., 1991 \\
\hline Daphnia carinata & $\mathrm{K}_{2} \mathrm{Cr}_{2} \mathrm{O}_{7}$ & $\operatorname{NOEC}(r) 14 j$ & 0,05 & Hickey, 1989 \\
\hline \multirow[t]{7}{*}{ Daphnia magna } & $\mathrm{K}_{2} \mathrm{Cr}_{2} \mathrm{O}_{7}$ & NOEC (r) $21 \mathrm{j}$ & 0,018 & Kuhn et al., 1989 \\
\hline & $\mathrm{K}_{2} \mathrm{Cr}_{2} \mathrm{O}_{7}$ & NOEC $(r) 21 \mathrm{j}$ & 0,035 & Sloof et Canton, 1983 \\
\hline & $\mathrm{K}_{2} \mathrm{Cr}_{2} \mathrm{O}_{7}$ & NOEC (c) 21 j & 0,060 & Van Leeuwen et al., 1987 \\
\hline & $\mathrm{K}_{2} \mathrm{Cr}_{2} \mathrm{O}_{7}$ & $\operatorname{NOEC}(r) 21 \mathrm{j}$ & 0,350 & Van Leeuwen et al., 1987 \\
\hline & $\mathrm{K}_{2} \mathrm{Cr}_{2} \mathrm{O}_{7}$ & NOEC (r) $14 \mathrm{j}$ & 0,025 & Hickey, 1989 \\
\hline & $\mathrm{Na}_{2} \mathrm{Cr}_{2} \mathrm{O}_{7}$ & NOEC (r) $14 \mathrm{j}$ & 0,0005 & Elnabarawy et al., 1986 \\
\hline & $\mathrm{K}_{2} \mathrm{Cr}_{2} \mathrm{O}_{7}$ & & 0,019 & Moyenne géométrique (r) \\
\hline
\end{tabular}




\begin{tabular}{|c|c|c|c|c|}
\hline Espèce & $\begin{array}{c}\text { substance } \\
\text { testée }\end{array}$ & Critère d'effet & $\begin{array}{c}\mathrm{Cr} \\
\mathrm{mg} / \mathrm{L}\end{array}$ & Référence \\
\hline \multicolumn{5}{|l|}{ Cœlentérés } \\
\hline Hydra littoralis & $\mathrm{K}_{2} \mathrm{Cr}_{2} \mathrm{O}_{7}$ & $\operatorname{NOEC}(r) 11 \mathrm{j}$ & 0,035 & Dannenberg, 1984 \\
\hline Hydra oligactis & $\mathrm{K}_{2} \mathrm{Cr}_{2} \mathrm{O}_{7}$ & $\operatorname{NOEC~(c)~} 21 \mathrm{j}$ & 1,1 & Sloof et Canton, 1983 \\
\hline \multicolumn{5}{|l|}{ Insectes } \\
\hline Culex pipiens & $\mathrm{K}_{2} \mathrm{Cr}_{2} \mathrm{O}_{7}$ & NOEC (dev) $25 \mathrm{j}$ & 1,1 & Sloof et Canton, 1983 \\
\hline \multicolumn{5}{|l|}{ Mollusques } \\
\hline Lymnaea stagnalis & $\mathrm{K}_{2} \mathrm{Cr}_{2} \mathrm{O}_{7}$ & $\begin{array}{l}\text { NOEC (r) } 40 \mathrm{j} \\
\text { NOEC (ecl) } 7 \mathrm{j}\end{array}$ & $\begin{array}{l}\mathbf{0 , 1 1} \\
0,35\end{array}$ & $\begin{array}{l}\text { Sloof et Canton, } 1983 \\
\text { Sloof et Canton, } 1983\end{array}$ \\
\hline \multicolumn{5}{|l|}{ Poissons } \\
\hline Catastomus commersoni & $\mathrm{Na}_{2} \mathrm{Cr}_{2} \mathrm{O}_{7}$ & NOEC (c) (60 j) & 0,29 & Sauter et al., 1976 \\
\hline Esox lucius & $\mathrm{Na}_{2} \mathrm{Cr}_{2} \mathrm{O}_{7}$ & NOEC (s) (20 j) & 0,538 & Sauter et al., 1976 \\
\hline Ictalurus punctatus & $\mathrm{Na}_{2} \mathrm{Cr}_{2} \mathrm{O}_{7}$ & $\begin{array}{c}\operatorname{NOEC}(\mathrm{c})(30 \mathrm{j}) \\
\operatorname{NOEC}(\mathrm{c})(30-60 \mathrm{j}) \\
\end{array}$ & $\begin{array}{l}\mathbf{0 , 1 5} \\
0,305\end{array}$ & $\begin{array}{l}\text { Sauter et al., } 1976 \\
\text { Sauter et al., } 1976\end{array}$ \\
\hline \multirow[t]{2}{*}{ Oncorhynchus mykiss } & $\mathrm{Na}_{2} \mathrm{Cr}_{2} \mathrm{O}_{7}$ & $\begin{array}{l}\operatorname{NOEC~(c)~(60~j)~} \\
\operatorname{NOEC~(c)~(8~mois)~}\end{array}$ & $\begin{array}{c}0,051 \\
0,1\end{array}$ & $\begin{array}{l}\text { Sauter et al., } 1976 \\
\text { Benoit, } 1976\end{array}$ \\
\hline & & & 0,071 & Moyenne géométrique (c) \\
\hline Oryzias latipes & $\mathrm{K}_{2} \mathrm{Cr}_{2} \mathrm{O}_{7}$ & $\begin{array}{l}\operatorname{NOEC}(\mathrm{m})(40 \mathrm{j}) \\
\operatorname{NOEC}(\mathrm{c})(40 \mathrm{j})\end{array}$ & $\begin{array}{r}3,5 \\
35 \\
\end{array}$ & $\begin{array}{l}\text { Sloof et Canton, } 1983 \\
\text { Sloof et Canton, } 1983\end{array}$ \\
\hline \multirow[t]{2}{*}{ Pimephales promelas } & $\begin{array}{l}\mathrm{Na}_{2} \mathrm{CrO}_{4} \\
\mathrm{Na}_{2} \mathrm{CrO}_{4} \\
\mathrm{Na}_{2} \mathrm{CrO}_{4} \\
\mathrm{Na}_{2} \mathrm{CrO}_{4} \\
\mathrm{Na}_{2} \mathrm{CrO}_{4} \\
\mathrm{Na}_{2} \mathrm{Cr}_{2} \mathrm{O}_{7}\end{array}$ & 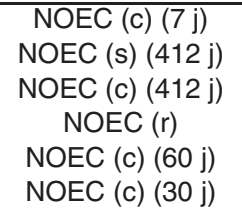 & $\begin{array}{c}1,1 \\
1 \\
3,95 \\
>3,95 \\
1 \\
0,05\end{array}$ & $\begin{array}{l}\text { De Graeve et al., } 1991 \\
\text { Pickering, } 1980 \\
\text { Pickering, } 1980 \\
\text { Pickering, } 1980 \\
\text { Pickering, } 1980 \\
\text { Broderius et Smith Jr., } 1979\end{array}$ \\
\hline & & & 0,68 & Moyenne géométrique (c) \\
\hline Poecilia reticulata & $\mathrm{K}_{2} \mathrm{Cr}_{2} \mathrm{O}_{7}$ & $\operatorname{NOEC~(c)~(28~j)~}$ & 3,5 & Sloof et Canton, 1983 \\
\hline Salvelinus fontinalis & $\mathrm{Na}_{2} \mathrm{Cr}_{2} \mathrm{O}_{7}$ & NOEC (c) (8 mois) & 0,01 & Benoit, 1976 \\
\hline Salvelinus namaycush & $\mathrm{Na}_{2} \mathrm{Cr}_{2} \mathrm{O}_{7}$ & $\operatorname{NOEC~(c)~(60~j)~}$ & 0,105 & Sauter et al., 1976 \\
\hline \multicolumn{5}{|l|}{ Batraciens } \\
\hline Xenopus laevis & $\mathrm{K}_{2} \mathrm{Cr}_{2} \mathrm{O}_{7}$ & $\begin{array}{l}\operatorname{NOEC}(\mathrm{c})(100 \mathrm{j}) \\
\operatorname{NOEC}(\mathrm{m})(100 \mathrm{j})\end{array}$ & $\begin{array}{c}1,1 \\
0,35 \\
\end{array}$ & Sloof et Canton, 1983 \\
\hline
\end{tabular}

$\mathrm{b}$ : biomasse, $\mathrm{c}$ : croissance, $\mathrm{r}$ : reproduction, $\mathrm{s}$ : suivie, $\mathrm{m}$ : mortalité

b) Organismes marins

\begin{tabular}{|l|c|c|c|l|}
\hline Espèce & substance testée & Critère d'effet & $\begin{array}{c}{[\mathbf{C r V I}]} \\
\mathbf{m g} / \mathbf{l}\end{array}$ & Référence \\
\hline Invertébrés & & & & \\
\hline Artemia salina & $\mathrm{Na}_{2} \mathrm{CrO}_{4}$ & NOEC & 7,11 & Kissa et al., 1984 \\
\hline Barentsia matsushimana & $\mathrm{K}_{2} \mathrm{Cr}_{2} \mathrm{O}_{7}$ & LOEC $(49 \mathrm{j})$ & 0,013 & Scholz, 1987 \\
\hline Cancer anthonyi & $\mathrm{K}_{2} \mathrm{Cr}_{2} \mathrm{O}_{7}$ & LOEC $(7 \mathrm{j})$ & 0,01 & Macdonald et al., 1988 \\
\hline Capitella capitata & $\mathrm{K}_{2} \mathrm{Cr}_{2} \mathrm{O}_{7}$ & NOEC $(5$ mois $)$ & 0,05 & Reish, 1977 \\
\hline Carcinus maenas & $\mathrm{Na}_{2} \mathrm{CrO}_{4}$ & NOEC $(12 \mathrm{j})$ & 40 & Raymont et Shields, 1963 \\
\hline Mysidopsis bahia & $\mathrm{K}_{2} \mathrm{Cr}_{2} \mathrm{O}_{7}$ & LOEC $(38 \mathrm{j})$ & 0,132 & Lussier et al., 1985 \\
\hline Neanthes arenaceodentata & $\mathrm{K}_{2} \mathrm{Cr}_{2} \mathrm{O}_{7}$ & NOEC $(440 \mathrm{j})$ & 0,013 & Oshida et al., 1981 \\
\hline Neanthes arenaceodentata & $\mathrm{K}_{2} \mathrm{Cr}_{2} \mathrm{O}_{7}$ & LOEC $(350 \mathrm{j})$ & 0,0125 & Mearns et al., 1976 \\
\hline Palaemon elegans (larves) & $\mathrm{Na}_{2} \mathrm{CrO}_{4}$ & NOEC $(36 \mathrm{j})$ & 0,52 & Van der Meer et al., 1988 \\
\hline Palaemonetes varians & $\mathrm{Na}_{2} \mathrm{CrO}_{4}$ & NOEC $(28 \mathrm{j})$ & 5,2 & Van der Meer et al., 1988 \\
\hline Palaemonetes varians (larves $)$ & $\mathrm{Na}_{2} \mathrm{CrO}_{4}$ & NOEC $(30 \mathrm{j})$ & 5,2 & Van der Meer et al., 1988 \\
\hline
\end{tabular}




\begin{tabular}{|l|c|c|c|l|}
\hline Espèce & substance testée & Critère d'effet & $\begin{array}{c}{[\mathbf{C r V I}]} \\
\mathbf{m g} / \mathbf{l}\end{array}$ & Référence \\
\hline Palaemonetes varians (larves) & $\mathrm{Na}_{2} \mathrm{CrO}_{4}$ & LOEC $(32 \mathrm{j})$ & 0,312 & Van der Meer et al., 1988 \\
\hline Praunus flexuosus & $\mathrm{Na}_{2} \mathrm{CrO}_{4}$ & NOEC $(23 \mathrm{j})$ & 1,0 & Van der Meer et al., 1988 \\
\hline Tisbe holothuriae & $\mathrm{Na}_{2} \mathrm{CrO}_{4}$ & LOEC & 0,5 & Apostolopoulou, 1981 \\
\hline Algues & & & & \\
\hline Microcystis pyritera & $\mathrm{K}_{2} \mathrm{Cr}_{2} \mathrm{O}_{7}$ & LOEC $(5 \mathrm{j})$ & 1,0 & Bernhard et Zattera, 1975 \\
\hline Skeletonema costatum & $\mathrm{K}_{2} \mathrm{Cr}_{2} \mathrm{O}_{7}$ & NOEC $(120 \mathrm{~h})$ & 0,35 & Cowgill et al., 1989 \\
\hline Thalassiosira pseudonana & $\mathrm{K}_{2} \mathrm{Cr}_{2} \mathrm{O}_{7}$ & NOEC & 0,104 & Thomas et al., 1980 \\
\hline
\end{tabular}

\section{Chrome III}

\begin{tabular}{|c|c|c|c|c|c|}
\hline & Espèce & $\begin{array}{c}\text { Substance } \\
\text { testée }\end{array}$ & $\begin{array}{l}\text { Critère } \\
\text { d'effet }\end{array}$ & $\begin{array}{c}\mathrm{Cr} \\
\mathrm{mg} / \mathrm{l}\end{array}$ & Référence \\
\hline \multirow[t]{3}{*}{ Poissons } & $\begin{array}{l}\text { Brachydanio rerio } \\
\text { (ELS) }\end{array}$ & $\mathrm{Cr}(\mathrm{OH}) \mathrm{SO}_{4}$ & NOEC (30 j) & $>\quad 313$ & IUCLID, 1999 \\
\hline & $\begin{array}{l}\text { Oncorhynchus } \\
\text { mykiss (ELS) }\end{array}$ & $\mathrm{Cr}\left(\mathrm{NO}_{3}\right)_{3}$ & NOEC & 0,05 & $\begin{array}{l}\text { Stevens et Chapman, } \\
1984\end{array}$ \\
\hline & Pimephales promelas & $\mathrm{CrK}\left(\mathrm{SO}_{4}\right)_{2}$ & NOEC (cdv) & $>0,05$ & Pickering (unpublished) \\
\hline \multirow[t]{3}{*}{ Invertébrés } & Daphnia magna & $\mathrm{Cr}\left(\mathrm{NO}_{3}\right)_{3}$ & NOEC (cdv) & $\begin{array}{l}0,047- \\
0,129^{*}\end{array}$ & $\begin{array}{l}\text { Chapman et al. } \\
\text { (unpublished) }\end{array}$ \\
\hline & Daphnia magna & $\mathrm{CrCl}_{3}$ & $\operatorname{NOEC}(21 \mathrm{j})$ & 3,4 & Kühn et al., 1989 \\
\hline & $\begin{array}{l}\text { Neanthes } \\
\text { arenaceodentata }\end{array}$ & $\mathrm{CrCl}_{3}$ & NOEC & $>50,4$ & Oshida et al., 1976 \\
\hline \multirow[t]{2}{*}{ Algues } & \begin{tabular}{|l} 
Chlorella \\
pyrenoidosa
\end{tabular} & $\mathrm{CrK}\left(\mathrm{SO}_{4}\right)_{2}$ & NOEC (5 j) & $>2$ & $\begin{array}{l}\text { Meisch et Schmitt- } \\
\text { Beckmann, } 1979\end{array}$ \\
\hline & $\begin{array}{l}\text { Scenedesmus } \\
\text { subspicatus }\end{array}$ & $\mathrm{Cr}(\mathrm{OH}) \mathrm{SO}_{4}$ & $\operatorname{NOEC~(24~h)~}$ & $>0,313$ & IUCLID, 1999 \\
\hline
\end{tabular}

Note : * les effets dépendent de la dureté du milieu d'essai.

\section{II.2 Paramètres d'écotoxicité vis-à-vis des organismes du sédiment}

MacDonald et al. (2000) proposent une TEC de 43,4 mg/kg sédiment sec. $28 \%$ des sédiments étudiés par MacDonald et al. (2000) étaient toxiques bien que la concentration en chrome soit en dessous de la TEC. Par ailleurs, les valeurs ayant servi à la détermination de la TEC varient entre 26 et $80 \mathrm{mg} / \mathrm{kg}$.

\section{II.3 Paramètres d'écotoxicité vis-à-vis des organismes terrestres}

\section{Chrome VI}

Le chrome $\mathrm{VI}$ est rapidement transformé en chrome III dans le sol. Les résultats ci-dessous correspondent à des essais lors desquels du chrome VI a été testé et sont exprimés en mg de chrome total. 


\begin{tabular}{|c|c|c|c|c|}
\hline & Espèce & $\begin{array}{l}\text { Critère } \\
\text { d'effet }\end{array}$ & $\begin{array}{c}\text { Valeur (mg } \\
\text { Cr/kg sol sec) }\end{array}$ & Référence \\
\hline \multirow[t]{12}{*}{ Végétaux } & avoine & $\mathrm{CE}_{50}(14 \mathrm{j})$ & 30 & Guenther et Pestemer, 1990 \\
\hline & avoine & $\operatorname{NOEC}(14 \mathrm{j})$ & $3,5-11$ & Adema et Henzen, 1989 \\
\hline & avoine & $\mathrm{CE}_{50}(14 \mathrm{j})$ & $7,4-31$ & Adema et Henzen, 1989 \\
\hline & Taitue & $\operatorname{NOEC}(14 \mathrm{j})$ & $0,35->11$ & Adema et Henzen, 1989 \\
\hline & laitue & $\mathrm{CE}_{50}(14 \mathrm{j})$ & $1,8->11$ & Adema et Henzen, 1989 \\
\hline & tomates & $\operatorname{NOEC}(14 \mathrm{j})$ & $3,2-10$ & Adema et Henzen, 1989 \\
\hline & tomates & $\mathrm{CE}_{50}(14 \mathrm{j})$ & $6,8-21$ & Adema et Henzen, 1989 \\
\hline & navet & $\mathrm{CE}_{50}(10 \mathrm{j})$ & 8,25 & Guenther et Pestemer, 1990 \\
\hline & cresson & $\mathrm{CE}_{50}(3 \mathrm{j})$ & 30 & Guenther et Pestemer, 1990 \\
\hline & petits pois & LOEC (56 j) & 452 & Miller et al., 1980 \\
\hline & mais & NOEC (56 j) & 452 & Miller et al., 1980 \\
\hline & Ivraie & LOEC (30 j) & 50 & Otabbong, 1990 \\
\hline \multirow[t]{2}{*}{ Annélides } & Eisenia fetida & $\mathrm{CE}_{50}(14 \mathrm{j})$ & 792 & Cabridenc et al., 1984 \\
\hline & Enchytraeus albidus & $\mathrm{CL}_{50}(28 \mathrm{j})$ & 146 & $\begin{array}{l}\text { Römbke, 1989; Römbke } \\
\text { et Knacker, } 1989\end{array}$ \\
\hline Micro-organismes & Nitrification & $\operatorname{LOEC~(28~j)~}$ & 3,2 & Ueda et al., 1988 \\
\hline
\end{tabular}

Les essais de Guenther et Pestemer (1990) consistent en l'exposition de jeunes plants d'avoine et de navets à du chrome VI pendant 10-14 jours. L'effet sur le cresson est un effet sur la germination.

Les essais d'Adema et Henzen (1989) ont été conduits selon la ligne directrice OCDE 208 sur différents types de sol. Les expériences ont été conduites jusqu'à 14 jours après germination.

Dans l'essai de Miller et al. (1980), 452 mg/kg correspond à la plus petite concentration testée.

Chrome III

\begin{tabular}{|l|c|c|c|l|}
\hline & Espèce & Critère d'effet & $\begin{array}{c}\text { Valeur } \\
\text { (mg Cr/kg } \\
\text { sol sec) }\end{array}$ & Référence \\
\hline Végétaux & Blé & LOEC & 100 & Moulinier et Mazoyer, 1968 \\
\hline Annélides & Eisenia andrei & $\mathrm{NOEC}(21 \mathrm{j})$ & 32 & Van Gestel et al., 1993 \\
\hline Micro organismes & & $\mathrm{HC}_{5}(50 \%)$ & 5,9 & Crommentuijn et al., 1997 \\
\hline
\end{tabular}

\section{II.4 Effets sur les prédateurs}

Lors d'une étude expérimentale réalisée chez la souris, les animaux ont été exposés au chrome $\mathrm{VI}\left(\mathrm{K}_{2} \mathrm{CrO}_{4}\right)$ à des doses de 0 à $25 \mathrm{mg} / \mathrm{l}$ pendant 1 an dans l'eau de boisson. Une NOAEL de $20 \mathrm{mg} / \mathrm{kg} / \mathrm{j}$ a été définie pour le chrome VI. Le guide technique européen (C.E., 1996) propose des facteurs de conversion en fonction des espèces afin de se rapporter à une concentration dans la nourriture. Le rapport poids corporel sur le taux de prise de nourriture journalière proposé est de 8,3. D'où NOEC = $166 \mathrm{mg} / \mathrm{kg}$ de nourriture. 


\section{CONCENTRATIONS SANS EFFET PRÉVISIBLE POUR L'ENVIRONNEMENT (PNEC)}

\section{Chrome VI}

Étant donné qu'un nombre important de NOECs long-terme existe pour des micro-crustacés, des poissons et des algues, la PNEC peut être évaluée par la méthode statistique.

Les valeurs suivantes ont été obtenues avec les résultats d'essais vis-à-vis des organismes d'eaux douces en supposant que les données suivent une loi log logistique :

HC5 $(50 \%)=10,4 \mu \mathrm{g} / \mathrm{l}(\mathrm{HC} 5(95 \%)=4,1 \mu \mathrm{g} / \mathrm{l})$

(avec une loi log normale on obtient : HC5 = 11,5 $\mu \mathrm{g} / \mathrm{l}\left(\mathrm{IC} \_90 \%=[4,8 ; 21,9]\right)$ )

Toxicité long terme du chrome VI vis-à-vis des organismes d'eau douce

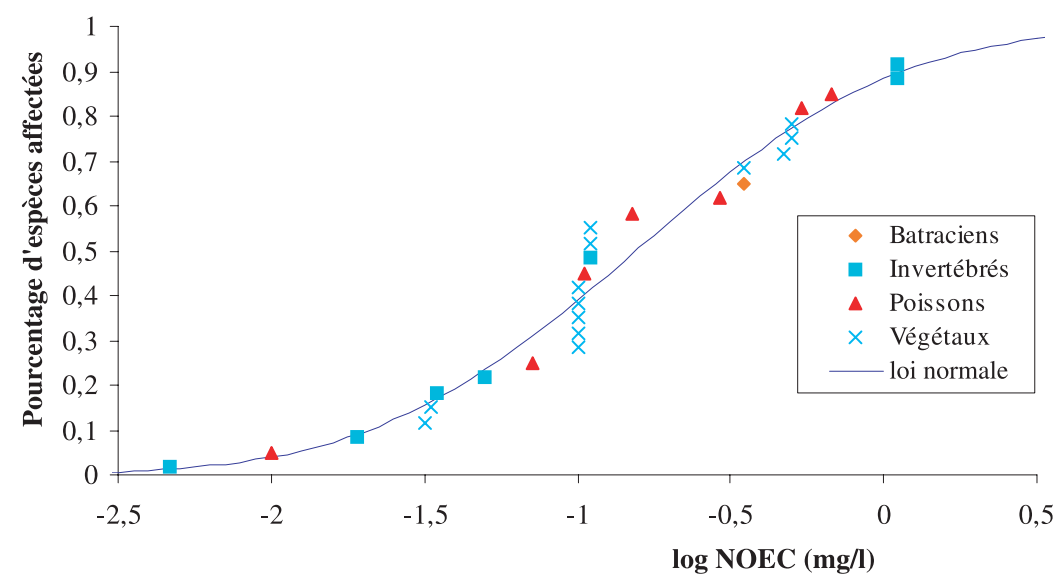

Le nombre de données est relativement important (28 espèces aquatiques d'eau douce). Cependant, il n'existe qu'un résultat vis-à-vis des insectes et des mollusques. Par ailleurs, le résultat vis-à-vis de Ceriodaphnia dubia est inférieur à la HC5. Or, cette valeur est la moyenne géométrique de 18 résultats d'essai lors d'un essai circulaire. Elle ne peut en conséquence être ignorée. C.E. (2001) suggère de prendre pour PNEC la borne inférieure de l'intervalle de confiance autour de la HC5. D'où :

\section{PNECeau douce $(\mathrm{CrVI})=4,1 \mu \mathrm{g} / \mathrm{l}$.}

En ce qui concerne le milieu marin, il n'est pas possible de dériver une PNEC « marine » uniquement à partir des résultats d'essai vis-à-vis des organismes marins car il n'existe pas de résultats vis-à-vis de poissons marins. La figure suivante montre la distribution de l'ensemble des résultats vis-à-vis des organismes aquatiques. 
Les valeurs suivantes ont été calculées à l'aide de l'ensemble des données aquatiques présentées ci-dessus en supposant que les données suivent une loi log normale :

$\mathrm{HC} 5=7,8 \mu \mathrm{g} / \mathrm{l}\left(\mathrm{IC} \_90 \%=[3,4 ; 15,2]\right)$

Toxicité long terme du chrome VI vis-à-vis des organismes aquatiques

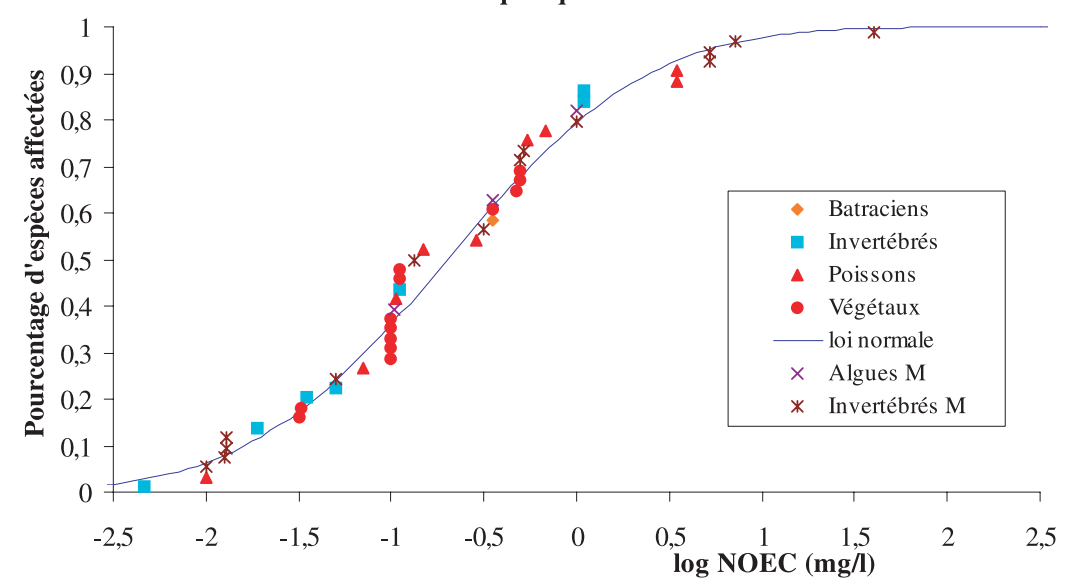

En utilisant le même raisonnement que pour l'eau douce on obtient :

PNECeau marine $(\mathrm{CrVI})=3,4 \mu \mathrm{g} / \mathrm{l}$.

Pour le sol, la plus faible NOEC observée est de $0,35 \mathrm{mg} / \mathrm{kg}$ pour les plantes. II existe des données long terme pour des plantes, des invertébrés (bien qu'il n'existe pas de réelle NOEC pour les invertébrés, les données disponibles montrent qu'ils ne sont pas plus sensibles que les autres espèces) et les microorganismes. Un facteur d'extrapolation de 10 peut en conséquence être utilisé pour dériver la PNEC. D'où :

\section{PNECsol $=0,035 \mathrm{mg} / \mathrm{kg}$ sol sec $=0,031 \mathrm{mg} / \mathrm{kg}$ sol humide .}

Une PNEC par empoisonnement secondaire (PNEC oral) peut être estimée avec un facteur d'extrapolation de 10 sur la NOEC pour mammifère.

D'où :

\section{PNECprédateurs = 17 mg/kg de nourriture.}

Chrome III

II existe des données chroniques sur trois niveaux trophiques, mais pas suffisamment pour utiliser une méthode d'extrapolation statistique de la PNEC. II est donc possible d'utiliser un facteur d'extrapolation de 10 sur la plus faible NOEC 
(C.E., 1996). D'où :

PNECeau douce $(\mathrm{Cr}$ III) $=4,7 \mu \mathrm{g} / \mathrm{l}$.

II n'y a pas de résultats d'essais sur organismes marins pour le Chrome III. Compte tenu de la spécificité du comportement du chrome en milieu marin, il ne serait pas réaliste de dériver une PNEC pour les organismes marins à partir des résultats d'essais effectués en eau douce.

Une PNEC peut être obtenue à partir de la TEC proposée par MacDonald et al. (2000) avec un facteur d'extrapolation de 20. D'où :

\section{PNECséd eau douce $=2,2 \mathrm{mg} / \mathrm{kg}$ sédiment $\mathrm{sec}$.}

Pour dériver la PNEC pour les sédiment marins, faute d'informations supplémentaires nous proposons en première approche d'utiliser la même valeur que pour les sédiments d'eau douce. D'où :

\section{PNECséd marin = 2,2 $\mathrm{mg} / \mathrm{kg}$ sédiment sec.}

Pour le compartiment terrestre, il existe des données long terme sur les trois niveaux trophiques. Une NOEC de $32 \mathrm{mg} \mathrm{Cr} / \mathrm{kg}$ est rapportée. En appliquant un facteur d'extrapolation de 10 sur cette valeur, on obtient une PNEC de 3,2 mg/kg sol sec. Cette valeur est inférieure à la HC5 rapportée par Crommentuijn (1997) sur les processus microbiens. D'où :

\section{PNECsol = 3,2 $\mathrm{mg} / \mathrm{kg}$ sol $\mathrm{sec}=2,8 \mathrm{mg} / \mathrm{kg}$ sol humide.}

Compte tenu de l'absence de données de toxicité par voie orale chez les mammifères pour le Chrome III, il n'est pas possible de déterminer une PNEC pour les prédateurs.

\section{BIBLIOGRAPHIE}

Adema D.M.M. and Henzen L. (1989) - A comparison of plant toxicities of some industrial chemicals in soil culture and soilless culture. Exotox. Environ. Safety., 18, 219-229.

Apostolopoulou (1981) - cité dans E.C. (2001).

Benoit D.A. (1976) - Toxic effects of hexavalent chromium on brook trout (Salvelinus fontinalis) and rainbow trout (Salmo gairdneri). Water Res., 10, 497-500.

Bernhard M. and Zattera A. (1975) Major pollutants in the marine environment. vol. , In : Marine Pollution and Marine Waste Disposal., E. A. Pearson and E. D. Frangipane Eds, 195.

Broderius S.J. and Smith L.L.J. (1979) - Lethal and sublethal effects of binary mixtures of cyanide and hexavalent chromium, zinc, or ammonia to the fathead minnow, Pimephales promelas, and rainbow trout, Salmo gairdneri. J. Fish. Res. Board Can., $36,2,164-172$.

Cabridenc R., Chambon A., Ducros M. and Lepailleur H. (1984) Influence du substrat sur la toxicité des substances chimiques vis-à-vis des vers des terre. In : Actes du Symposium International sur l'Ecotoxicologie Terrestre, Eds, 199-214. 
Cairns J.J., Buikema A.L.J., Heath A.G. and Parker B.C. (1978) - Effects of temperature on aquatic organism sensitivity to selected chemicals. Virginia Water Resources Research Center. Blacksburg, Virginia.

Calamari D., Gaggino G.F. and Pacchetti G. (1982) - Toxicokinetics of low levels of Cd, Cr, $\mathrm{Ni}$ and their mixture in long-term treatment on Salmo gairdneri Rich. Chemosphere, 11, 59-70.

Chapman G.A. et al. (unpublished) - Effects of water hardness on the toxicity of metals to Daphnia magna. US-EPA. Corvallis, Oregon.

Christensen E.R., Chen C.-Y. and Kroeger S.R. (1983) Algal growth under single and multiple toxicant stress. In : Heavy Metals in the Environment. 4th International Conference., Eds, 662-665.

C.E. (1996) - Technical guidance document in support of Commission Directive 93/67/EEC on risk assessment for new notified substances and Commission Regulation (EC) $\mathrm{N}^{\circ}$ $1488 / 94$ on risk assessment for existing substances. Office for Official Publications of the EC. Luxembourg.

C.E. (2001) - Chromates. Evaluation des risques dans le cadre du règlement CE 793/93 sur les substances existantes (projet mai 2001).

Cowgill U.M., Milazzo D.P. and Landenberger B.D. (1989) - Toxicity of Nine Benchmark Chemicals to Skeletonema costatum, a Marine Diatom. Environ. Toxicol. Chem., 8, 5, 451-455.

Crommentuijn T., Polder M.D. and Van de Plassche E.J. (1997) - Maximum permissible concentrations and negligible concentrations for metals, taking background concentrations into account. RIVM. 601501001.

Dannenberg R. (1984) - Effahrungen mit einem limnischen Hydroidentest. Z. WasserAbwasser-Forsch., 17, 16-19.

DeGraeve G.M., Cooney J.D., McIntyre D.O., Polluck T.L., Reichenbach N.G. and Marcus M.D. (1991) - Variability in the performance of the seven-day fathead minnow (Pimephales promelas) larval survival and growth test : an intra-and interlaboratory study. Environ. Tox. Chem., 10, 1189-1203.

Elnabarawy M.T., Welter A.N. and Robideau R.R. (1986) - Relative sensitivity of three Daphnia species to selected organic and inorganic chemicals. Environmental Toxicology and Chemistry, 5, 393-398.

Guenther P. and Pestemer W. (1990) - Environ. Manage., 14, 381-388.

Giesy J.J.P. and Wiener J.G. (1977) - Frequency distribution of trace metal concentrations in five freshwater fishes. Trans. Amer. Fish. Soc., 106, 393-403.

Hernandez F., Diaz J., Medina J., Del Ramo J. and Pastor A. (1986) - Determination of chromium in treated crayfish, Procambarus clarkii, by electrothermal AAS : study of chromium accumulation in different tissues. Bull. Environ. Contam. Toxicol., 36, 851857.

Hickey C.W. (1989) - Sensitivity of four New Zealand cladoceran species and Daphnia magna to aquatic toxicants. New Zealand J. Marine Fresh. Res., 23, 131-137.

HSDB (1999) - Hasardous Substances Data Bank, National Library of Medicine.

I.A.E.A. (1985) - Sediments Kds and concentration factors for radionuclides in the marine environment. International Atomic Energy Agency. Technical Report Series, No. 247.

IUCLID (1999) - International Uniform Chemical Information Database.

Jouany et al. (1982) - cité dans E.C. (2001).

Kissa E., Moraitou-Apostolopoulou M. and Kiortsis V. (1984) - Effects of four heavy metals on survival and hatching rate of Artemia salina (L.). Arch. Hydrobiol., 100, 255-264. 
Kühn R., Pattard M., Pernak K.D. and Winter (1989) - Results of the harmful effects of water pollutants to Daphnia magna in the 21 day reproduction test. Wat. Res., 23, 4, 501-510.

Kühn R. and Pattard M. (1990) - Results of the harmful effects of water pollutants to green algae (Scenedesmus subspicatus) in the cell multiplication inhibition test. Wat. Res., 24, 1, 31-38.

Lussier S.M., Gentile J.H. and Walker J. (1985) - Acute and chronic effects of heavy metals and Cyanide on Mysidopsis bahia (Crustacea : Mysidacea). Aquat. Toxicol., 7, 1/2, 2535.

Macdonald J.M., Shields J.D. and Zimmer-Faust R.K. (1988) - Acute toxicities of eleven metals to early life-history stages of the yellow crab Cancer anthonyi. Marine Biol., 98, 201-207.

MacDonald D.D., Ingersoll C.G. and Berger T.A. (2000) - Development and evaluation of consensus-based sediment quality guidelines for freshwater ecosystems. Archives of Environmental Contamination and Toxicology, 39, 20-31.

Mearns A.J., Oshida P.S., Sherwood M.J., Young D.R. and Reish D.J. (1976) - Chromium effects on coastal organisms. J. Water Pollut. Control Fed., 48, 1929-1938.

Meisch H.-U. and Schmitt-Beckmann I. (1979) - Influence of tri and hexavalent chromium on two Chlorella strains. Z. Pflanzenphysiol. Bd., 94, 231-239.

Miller R.W., Honarvar S. and Hunsaket B. (1980) - Effects of drilling fluids on soils and plants : I. Individual fluid components. J. Environ. Qual., 9, 547-552.

Moulinier H. and Mazoyer R. (1968) - Contribution à l'étude de l'action du chrome sur la croissance des végétaux. Ann. Agron., 19, 553-567.

Nyholm N. (1991) - Toxic effects on algal phosphate uptake. Environ. Toxicol. Chem., 10, 581-584.

Oshida P.S. et al. (1976) - The effects of hexavalent and trivalent chromium on Neanthes arenaceodentata (Polychaete : Annelida). S. California Coastal Water Res. Proj. El Segundo, California.

Oshida P.S., Word L.S. and Mearns A.J. (1981) - Effects of hexavalent and trivalent chromium on the reproduction of Neanthes arenaceodentata (Polychaeta). Marine Environ. Res., 5, 41-49.

Otabbong E. (1990) - Chemistry of $\mathrm{Cr}$ in some Swedish soils. V. Interaction between $\mathrm{CrO}_{3}$ and $\mathrm{Si}(\mathrm{OH})_{4}$ and its impact on Cr toxicity and elemental contents in ryegrass (Loium perenne). Plant Soil, 123, 89-93.

Pickering Q.H. (1980) - Chronic toxicity of hexavalent chromium to the fathead minnow (Pimephales promelas). Arch. Environ. Contam. Toxicol., 9, 405-413.

Pickering Q.H. (unpublished) - Chronic toxicity of trivalent chromium to the fathead minnow (Pimephales promelas) in hard water. As quoted in US-EPA (1985).

Raymont J.E.G. and Shields J. (1963) - Toxicity of copper and chromium in the marine environment. Int. J. Air Water Pollut., 7, 435-443.

Reish D.J. (1977) Effects of chromium on the life history of Capitella capitata (Annelidea : Polychaeta). vol. , In : Physiological Responses of Marine Biota to Pollutants., Eds, 199-207.

RIVM (1999) - Environmental Risk Limits in the Netherlands. RIVM. 601640001.

Römbke J. (1989) - Enchytraeus albidus (Enchytraeidae, Oligochaeta) as a test organism in terrestrial laboratory systems. Arch. Toxicol. Suppl., 13, 402-405.

Römbke J. and Knacker T. (1989) - Aquatic toxicity test for enchytraeids. Hydrobiologia, $180,235-242$. 
Sauter S., Buxton K.S., Macek K.J. and Petrocelli S.R. (1976) - Effects of exposure to heavy metals on selected freshwater fish. US-EPA, Aquatic Toxicology Lab. Wareham, Mass.

Scholz N. (1987) - Barentsia matsushimana, a marine entoproct suitable for bioassays. Bull. Environ. Contam. Toxicol., 38, 634-640.

Slooff W. and Canton J.H. (1983) - Comparison of the susceptibility of 11 freshwater species to 8 chemical compounds. II. (semi)chronic toxicity tests. Aquat. Toxicol., 4, 271-281.

Staves R.P. and Knaus R.M. (1985) - Chromium removal from water by three species of duckweeds. Aquat. Botany, 23, 261-273.

Stevens D.G. and Chapman G.A. (1984) - Toxicity of trivalent chromium to early life stage of steelhead trout. Environ. Toxicol. Chem., 5, 125.

Thomas W.H., Hollibaugh J.T. and Seibert D.L.R. (1980) - Effects of heavy metals on the morphology of some marine phytoplankton. Phycologia, 19, 202-209.

Ueda K., Kobayashi M. and Takahashi E. (1988) - Effect of chromate and organic amendments on the composition and activity of microorganism flora in soil. Soil Sci. Plant Nutr., 34, 233-240.

US-EPA (1980) - Ambient water quality criteria for chromium. United States Environmental Protection Agency. EPA 440/5-80-006.

Van der Meer C., Teunissen C. and Boog T.F.M. (1988) - Toxicity of sodium chromate and 3,4-Dichloroaniline to crustaceans. Bull.Environ. Contam. Toxicol., 40, 204-211.

Van Gestel C.A.M., Dirven-van Breemen E.M. and Baerselman R. (1993) - Accumulation and elimination of cadmium, chromium and zinc and effects on growth and reproduction in Eisenia andrei (Oligochaeta, Annelida). Sci. Total Environ., Suppl. I, 585-597.

Van Leeuwen C.J., Niebeek G. and Rijkevoer M. (1987) - Effects of chemical stress on the population dynamics of Daphnia magna: a comparison of two test procedures. Ecotox. Environ. Safety, 14, 1-11.

Van Weerelt M., Pfeiffer W.C. and Fiszman M. (1984) - Uptake and release of ${ }^{51} \mathrm{Cr}(\mathrm{VI})$ and ${ }^{51} \mathrm{Cr}(\mathrm{III})$ by barnacles (Balanus sp.). Marine Environ. Res., 11, 201-21. 